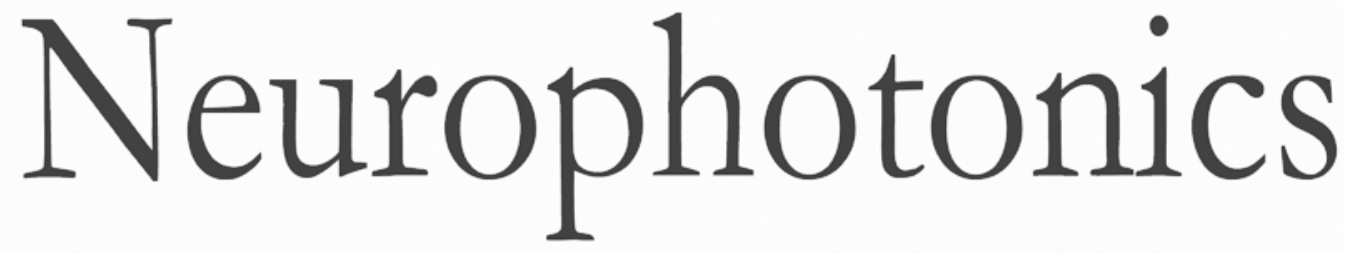

\title{
Functional near-infrared spectroscopy in movement science: a systematic review on cortical activity in postural and walking tasks
}

Fabian Herold

Patrick Wiegel

Felix Scholkmann

Angelina Thiers

Dennis Hamacher

Lutz Schega 


\title{
Functional near-infrared spectroscopy in movement science: a systematic review on cortical activity in postural and walking tasks
}

\author{
Fabian Herold, ${ }^{a, \star}$ Patrick Wiegel, ${ }^{b}$ Felix Scholkmann, ${ }^{c}$ Angelina Thiers, ${ }^{a}$ Dennis Hamacher, ${ }^{a}$ and Lutz Schega \\ ${ }^{a}$ Otto von Guericke University Magdeburg, Institute III, Department of Sport Science, Magdeburg, Germany \\ bUniversity of Freiburg, Department of Sport Science, Freiburg, Germany \\ 'University of Zurich, University Hospital Zurich, Department of Neonatology, Biomedical Optics Research Laboratory, Zurich, Switzerland
}

\begin{abstract}
Safe locomotion is a crucial aspect of human daily living that requires well-functioning motor control processes. The human neuromotor control of daily activities such as walking relies on the complex interaction of subcortical and cortical areas. Technical developments in neuroimaging systems allow the quantification of cortical activation during the execution of motor tasks. Functional near-infrared spectroscopy (fNIRS) seems to be a promising tool to monitor motor control processes in cortical areas in freely moving subjects. However, so far, there is no established standardized protocol regarding the application and data processing of fNIRS signals that limits the comparability among studies. Hence, this systematic review aimed to summarize the current knowledge about application and data processing in fNIRS studies dealing with walking or postural tasks. Fifty-six articles of an initial yield of 1420 publications were reviewed and information about methodology, data processing, and findings were extracted. Based on our results, we outline the recommendations with respect to the design and data processing of fNIRS studies. Future perspectives of measuring fNIRS signals in movement science are discussed. ๑ The Authors. Published by SPIE under a Creative Commons Attribution 3.0 Unported License. Distribution or reproduction of this work in whole or in part requires full attribution of the original publication, including its DOI. [DOI: 10.1117/1.NPh.4.4.041403]
\end{abstract}

Keywords: functional near-infrared spectroscopy; optical neuroimaging; motor control; walking; posture.

Paper 17031SSRRR received Mar. 4, 2017; accepted for publication Jun. 23, 2017; published online Aug. 1, 2017.

\section{Introduction}

Safe locomotion is indispensable for human daily living and requires good functionality of motor control processes. The efficiency of motor control processes of daily motor activities such as walking ${ }^{1,2}$ and standing ${ }^{3,4}$ relies on complex neuronal networks encompassing subcortical and cortical brain structures. Studies show that a smaller gray matter volume is associated with lower gait performance indicated by increased gait variability $^{5-7}$ or slower gait velocity. ${ }^{8,9}$ Moreover, lower wholebrain gray matter volume goes along with worse postural balance performance irrespective of age, ${ }^{10}$ whereas the increase of gray matter volume is associated with balance improvements. ${ }^{1-13}$ In older age, however, shrinking of those cortical structures ${ }^{14,15}$ might diminish motor control capabilities. ${ }^{16}$ The substantial body of literature suggests that cortical structures play an important role for the motor control of daily motor tasks. Therefore, the assessment of cortical activity while subjects are moving is a key factor to foster a better understanding of neuromotor control which, in turn, could help to improve rehabilitation strategies. ${ }^{17}$

Brain activity can be measured by the following neuroimaging methods: functional magnetic resonance imaging (fMRI), magnetoencephalography (MEG), positron-emission-tomography (PET), electroencephalography (EEG), and functional near-infrared spectroscopy (fNIRS). While fMRI is considered as gold standard for the assessment of activity in cortical and subcortical structures, it suffers from the vulnerability for

*Address all correspondence to: Fabian Herold, E-mail: fabian.herold@st.ovgu de movement artifacts and the restricted range of motion in the scanner. ${ }^{18-21}$ Likewise, MEG exhibits a high vulnerability for motion artifacts ${ }^{18}$ while the use of PET does not allow repeated measurements due to the injection of radioactive tracers. ${ }^{20}$ EEG puts out not only signals with high temporal resolution but also signals with a relatively weak spatial resolution. ${ }^{18,22}$ Furthermore, EEG is vulnerable to artifacts, time consuming in preparation, ${ }^{18,22,23}$ and the signals are hard to interpret for nonexperts. ${ }^{24}$ Hence, fMRI, MEG, PET, and EEG suffer from specific restrictions that hamper a time-efficient evaluation of cortical activation in moving subjects.

fNIRS is a relatively new optical neuroimaging technique that uses the theory of neurovascular coupling. ${ }^{19,25-27}$ Neurovascular coupling results from the neuronal activity or glia activity that provokes an enhanced blood flow in an active brain region to satisfy energetic demands of the neuronal tissue. ${ }^{27-29}$ Based on these hemodynamic responses of neuronal cortical tissues, the fNIRS technology allows an indirect evaluation of brain activation (such as fMRI). ${ }^{18,19}$

Therefore, light with different wavelengths in the nearinfrared spectrum is emitted through the skull and undergoes some scattering and absorption processes inside the neuronal tissue. ${ }^{27,30,31}$ In the neuronal tissue, the chromophores such as oxygenated (oxyHb) and deoxygenated hemoglobin (deoxyHb) absorb light at different spectra ${ }^{19,20,32,33}$ whereas the nonabsorbed components of the scattered light leave the brain in a banana-shaped course. Those components are recorded by a detector on the head surface. ${ }^{30,31,34}$ Based on the described neurovascular coupling, an enhanced brain activation induces an intensified blood flow in the active brain regions leading to an increase in oxyHb and decrease of deoxyHb. ${ }^{27,30} \mathrm{As}$ 
a consequence of the different absorption spectra of the chromophores, the activity-dependent concentration changes in oxy- and deoxyHb can be quantified with the modified Beer-Lambert law and used as an indicator of regional brain activation. ${ }^{19,20,27,30,32}$

The advantage of optical neuroimaging using fNIRS is the possibility to measure cortical activity (quantified as changes in tissue oxygenation and blood perfusion, associated with neural activity) noninvasively ${ }^{25,27,35,36}$ with a relatively good spatial and temporal resolution. ${ }^{19-22}$ This benefit makes fNIRS systems suitable for the usage in special cohorts, such as children. ${ }^{18,20,22,36-40}$ Moreover, fNIRS systems are applicable even during outdoor activities ${ }^{41}$ and could be used as a monitoring tool in neurorehabilitation settings. ${ }^{18,42-44}$ From this point of view, fNIRS is a promising tool to understand the contribution of cortical areas in the neuromotor control of gross motor skills, such as posture and walking. ${ }^{17}$ However, the fNIRS technology also has some disadvantageous including a limited depth sensitivity that restricts the measurements of brain activity to cortical layers $^{36}$ and the vulnerability to systemic vascular changes that may contaminate the signal during strenuous physical tasks. ${ }^{27,45}$ In addition, no standardized procedures regarding the usage of fNIRS with respect to measuring cortical activity in moving subjects exist ${ }^{17,42}$ which clearly limits the comparability across existing studies.

This systematic review elucidates the application of fNIRS in neuromotor research and concentrates on two crucial motor tasks, namely locomotion and postural stability. In this context, we aim to give an overview about (a) the methodological approach of fNIRS and (b) the main findings of the fNIRS measurements reported in the literature.

\section{Systematic Literature Search and Data Extraction}

Two independent researchers performed a systematic literature search to identify all relevant studies applying fNIRS to investigate hemodynamic brain responses during walking and postural tasks on February 4, 2017. Therefore, we used the following search terms: gait OR walking OR posture OR "postural control" OR balance OR balancing OR sway AND NIRS OR fNIR OR fNIRS OR "functional near-infrared spectroscopy" OR "near-infrared spectroscopy" OR "functional near-infrared spectroscopic" OR "optical imaging system." All studies that used brain-computer interfaces, examined orthostatic regulation or animals, provided insufficient statistical methods, or used non-English language were excluded. During this procedure, six articles were excluded due to the lack of statistical analyses, ${ }^{46-48}$ ineligible measurement condition, ${ }^{49}$ and non-English language. ${ }^{50,51}$ The search and screening process is shown in Fig. 1. From the included studies, data about cohort characteristics, fNIRS methodology, and main findings were extracted.

\section{Results: Methodology Employed in the Studies}

In the following, we will provide information about the methodological approaches of the reviewed studies. We focused on general aspects regarding the application, data processing, and data analyzing of fNIRS (e.g., study design, used filter methods, and statistical analysis). Further information about the cohorts, tasks, sampling frequencies, wavelengths, and numbers of channels can be requested by e-mail from the corresponding author or is available in Ref. 52.

\subsection{Baseline Condition and Duration}

\subsubsection{Treadmill walking}

Fifteen studies investigating cortical activation during treadmill walking assessed baseline brain activation during quiet standing. ${ }^{53-67}$ In contrast, Eggenberger et al. ${ }^{68}$ chose slow walking $(2 \mathrm{~km} / \mathrm{h})$ for $1 \mathrm{~min}$ as the baseline condition. The duration of baseline cortical activation used for further analyses varied between $2.5^{55,56}$ and $20 \mathrm{~s}^{59-61}$ (for an overview see Table 1).

\subsubsection{Overground walking}

Twenty-one studies conducting overground walking, quantified baseline brain activation in a standing position. ${ }^{66,69-88}$ In contrast, two studies assessed baseline brain activation while walking ${ }^{89}$ or during a predefined time period prior to a freezing of gait event (FOG; a sudden, brief inability to start movement or to continue rhythmic, repeated movements despite the internal intention to move).$^{90}$ The duration to assess baseline brain activity ranged between $5 \mathrm{~s}^{69,73,76,81}$ and 5 min. ${ }^{78}$ Most studies used $10 \mathrm{~s}$ to quantify baseline brain activity. ${ }^{66,70-72,74,75,82-88}$ Interestingly, Holtzer et al. ${ }^{74,75,83-85}$ asked their participants to conduct a simple counting task (in steps of 1) during the baseline condition (for an overview see Table 1).

\subsubsection{Postural tasks}

In postural research, 13 studies assessed baseline brain activity during quiet standing. ${ }^{91-103}$ The temporal duration to quantify baseline brain activity ranged from $2^{93}$ to $60 \mathrm{~s} .^{97}$ In most studies, data of $30 \mathrm{~s}^{98-100}$ or a few seconds before starting the next trial $^{91,94,95,102}$ were used to assess baseline brain activation. In addition, Wang et al. ${ }^{104}$ used 20 min quiet sitting to measure baseline connectivity (for an overview see Table 1).

\subsection{Number and Duration of Trials and Rest Phases}

\subsubsection{Treadmill walking}

The studies that used a treadmill for the walking condition $^{53-68,105-107}$ are shown in Table 1. Per task, a minimum of 2 trials $^{105}$ and a maximum of 10 trials $^{54-56}$ were performed. Most studies used three to five trials to assess task-relevant cortical activity. ${ }^{53,57,59-62,64,64,65,67,106,107}$ Task phases were set to $30 \mathrm{~s}$ in the majority of the studies, ${ }^{55-61,65,68,105-107}$ but Harada et al., ${ }^{53}$ Kim et al., ${ }^{106}$ and Mihara et al. ${ }^{58}$ used $60 \mathrm{~s}$, Koenraadt et al. ${ }^{54}$ used $35 \mathrm{~s}$, Metzger et al. ${ }^{64}$ used $45 \mathrm{~s}$, Suzuki et al. ${ }^{67}$ used $40 \mathrm{~s}$, Suzuki et al. ${ }^{62}$ used $90 \mathrm{~s}$, and Fraser et al. ${ }^{63}$ used $120 \mathrm{~s}$. The time of the rest phases ranged in most studies between 25 and 60 s. ${ }^{53-57,59-62,65,68,106,107}$ Additionally, rest times of $15 \mathrm{~s}$ prior to $^{58,64}$ and after each walking trial $^{58}$ were reported while Suzuki et al. ${ }^{67}$ implemented 10 to $25 \mathrm{~s}$ between trials (for an overview see Table 1).

\subsubsection{Overground walking}

Twenty-three studies investigated cortical hemodynamic responses during overground walking. ${ }^{66,69-90}$ For each condition, $1,{ }^{70,79} 3,{ }^{76} 4,{ }^{87} 5,{ }^{66,69,71,77,80,81,86} 6{ }^{73-75,82-85}$ and 15 walks were used. ${ }^{89}$ Either the time for each task phase ranged between $10^{69}$ and $120 \mathrm{~s}^{66}$ or the participants were asked to walk a predetermined distance ranging between $\sim 4^{73-75,82,83,85}$ and $90 \mathrm{~m}^{86}$ The resting phases prior to and after each trial lasted $20 \mathrm{~s}^{77,79}$ or 


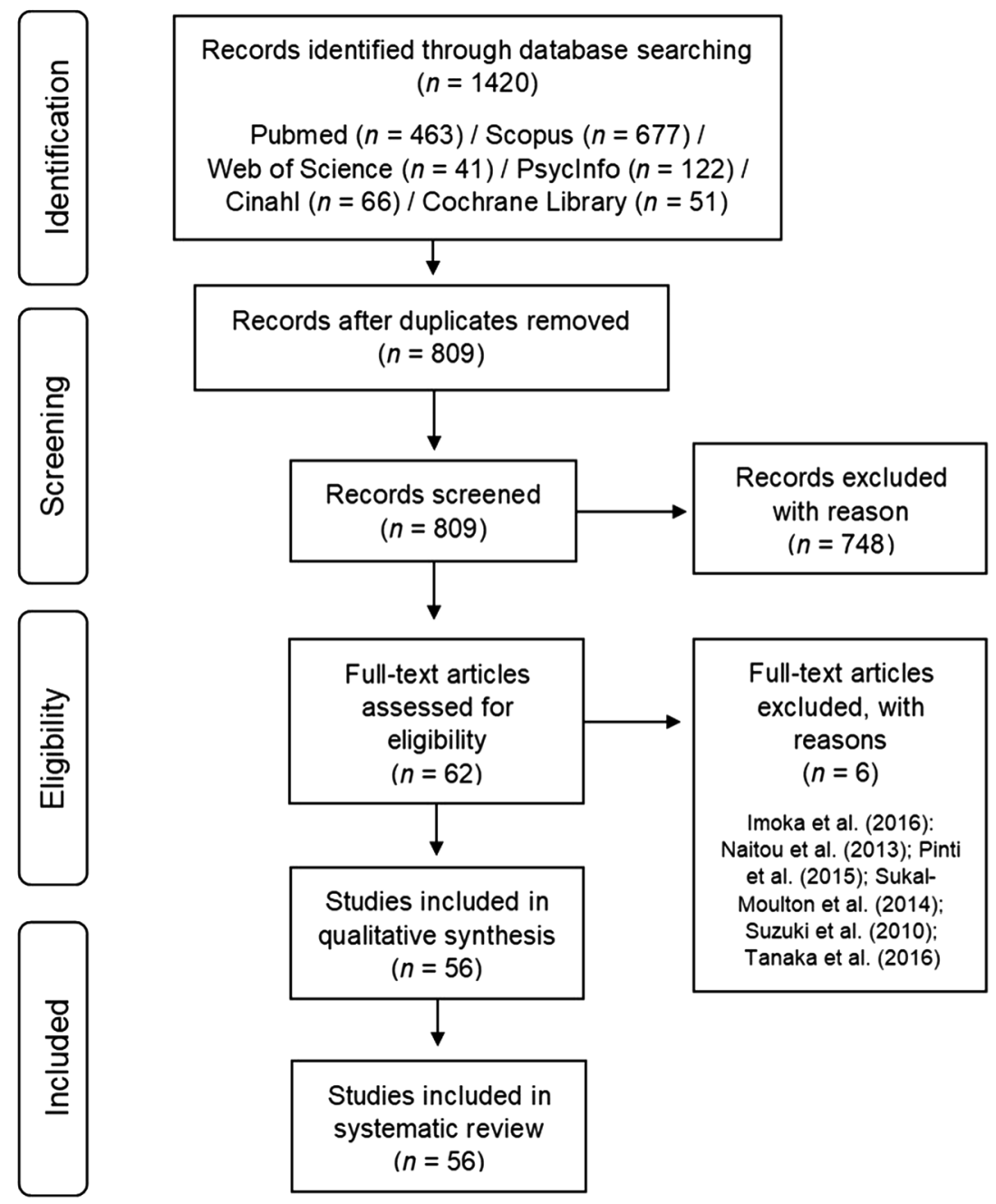

Fig. 1 Search process and identification of relevant studies.

$60 s^{76}$ and $10 s^{87}$ or $30 \mathrm{~s}$ between the trials. ${ }^{72}$ Two studies used $20 \mathrm{~s}$ of rest between successive trials and 1 to $2 \mathrm{~min}$ of rest between successive task blocks. ${ }^{80,81}$ Furthermore, in three studies, a rest of 2 min was used ${ }^{66,86,90}$ while one study allowed participants to rest $5^{88}$ or 30 min between tasks ${ }^{71}$ (for an overview see Table 1).

\subsubsection{Postural tasks}

Regarding the examination of brain activity during a sensory organization test (SOT; a balance test using quantitatively different visual, proprioceptive, and vestibular cues to assess the quality of postural stance stability), two trials, ${ }^{92}$ three trials,${ }^{96}$ or four trials were conducted ${ }^{103}$ which lasted $45,{ }^{92} 40,{ }^{103}$ or $20 \mathrm{~s}^{96}$ The participants of the three studies using mechanical perturbations performed $15^{95,102}$ to 30 trials $^{94}$ with a randomized perturbation duration of 5 to 20 s. $^{94,95,102}$ In semivirtual reality, seven trials with a task phase duration of $45 \mathrm{~s}$ were used. ${ }^{98}$ The rest between task phases depended on the conducted tasks (see Table 1) and ranged between 4 and $20 \mathrm{~s} .{ }^{91,94,95,101}$ In other studies, a rest of $1^{103}$ or 2 min was included..$^{92,98,99}$ To avoid fatigue, resting times after some trials that lasted a few minutes were common ${ }^{91,92,94}$ (for an overview see Table 1).

\subsection{Source-Detector Separation}

The closest distances between the optodes (source and detector) were reported to be $\sim 1 \mathrm{~cm}$, which was used as a short separation channel ${ }^{54}$ and was followed by an interoptode distance of $2.5 \mathrm{~cm}^{73-75,82-85,93,105}$ Three studies used $3.2 \mathrm{~cm},{ }^{91,92,97}$ and two studies used $3.5 \mathrm{~cm}^{80,81,90}$ Another seven studies used $4 \mathrm{~cm} .^{70,71,78-81,104}$ One study used a different distance between source and detector $(1,3 \text {, and } 4 \mathrm{~cm})^{54}$ and another one used multidistance measurement $(2.0,2.5,3.5$, and $4.0 \mathrm{~cm}) .{ }^{68}$ The remaining 36 studies set the interoptode distances at

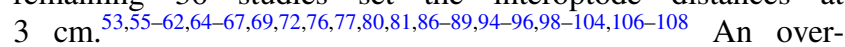
view on used source-detector is shown in Fig. 2(a).

\subsection{Placement of the Optodes}

The majority of studies used the international "10-20 EEG system" for the placement of the optodes. . $^{53-57,59-65,67-77,79,81-83,85,87-108}$ In some studies, an additional three-dimensional (3-D)digitizer was applied ${ }^{69,76,94-96,106,108}$ or an MRI scan was conducted $^{59-62,67,90,94-96,102,107}$ to coregister optode positions on the head. Other placement strategies (placing optodes on the forehead) were applied in four studies. ${ }^{58,66,80,86}$ 
Table 1 Overview about the population, study designs, and data processing steps of reviewed fNIRS studies (note that the number of trials is reported per condition).

\begin{tabular}{|c|c|c|c|}
\hline \multirow[t]{5}{*}{ First author } & $\begin{array}{l}\text { - Population } \\
\text { ( } n=\text { number of participants; } \\
\text { age in years } \pm \text { SD) }\end{array}$ & 1. Baseline condition & 1. DPF \\
\hline & \multirow[t]{4}{*}{ - Conditions } & 2. Baseline duration & 2. Data processing (filtering) \\
\hline & & $\begin{array}{l}\text { 3. Number of trials and } \\
\text { duration }\end{array}$ & 3. Final data processing \\
\hline & & 4. Rest phase duration & 4. Activation parameters \\
\hline & & & 5. Time used for analysis \\
\hline \multirow[t]{5}{*}{ Al-Yahya et al. ${ }^{65}$} & $\begin{array}{l}\text { - Stroke patients } \\
\quad(n=19 ; 59.61 \pm 15.03)\end{array}$ & 1. Quiet standing & $\begin{array}{l}\text { 1. Age-dependent value } \\
\left(4.99+0.067 \times \text { age }^{0.814}\right)\end{array}$ \\
\hline & $\begin{array}{l}\text { - Healthy old adults } \\
\quad(n=20 ; 54.35 \pm 9.38)\end{array}$ & $\begin{array}{l}\text { 2. } 25 \text { to } 45 \mathrm{~s} \text { (randomized } \\
\text { order) }\end{array}$ & 2. LPF at $0.67 \mathrm{~Hz}$ \\
\hline & \multirow[t]{3}{*}{$\bullet$ DTW vs. NW } & 3. $5 x ; 30 \mathrm{~s}$ & 3. Baseline correction; averaging \\
\hline & & \multirow{2}{*}{$\begin{array}{l}\text { 4. } 25 \text { to } 45 \mathrm{~s} \text { (between trials / } \\
\text { randomized order) }\end{array}$} & 4. Oxy- and deoxyHb \\
\hline & & & 5. 6 to $16 \mathrm{~s}$ after task begin \\
\hline \multirow[t]{5}{*}{ Atsummori et al. ${ }^{69}$} & $\begin{array}{l}\text { - Healthy young adults } \\
\quad(n=6 ; 29.7 \pm 3.3)\end{array}$ & 1. Quiet standing & 1. Constant value (no details reported) \\
\hline & \multirow[t]{4}{*}{ • DTW vs. NW } & 2. $5 \mathrm{~s}$ before task begin & 2. Not reported \\
\hline & & 3. $5 \times(\mathrm{DTW}) / 6 \times(\mathrm{NW}) ; 10 \mathrm{~s}$ & 3. Baseline correction; averaging \\
\hline & & 4. $20 \mathrm{~s}$ at beginning & 4. Oxy- and deoxyHb \\
\hline & & & 5. $6 \mathrm{~s}$ after task begin/ending \\
\hline \multirow[t]{5}{*}{ Basso-Moro et al. ${ }^{98}$} & $\begin{array}{l}\text { - Healthy young adults } \\
\quad(n=16 ; 29 \pm 4.8)\end{array}$ & 1. Quiet standing & $\begin{array}{l}\text { 1. Age-dependent value } \\
\left(4.99+0.067 \times \mathrm{age}^{0.814}\right)\end{array}$ \\
\hline & \multirow{4}{*}{$\begin{array}{l}\text { - Perturbations in semivirtual reality } \\
\text { with increasing difficulty }\end{array}$} & 2. Last $30 \mathrm{~s}$ (of $2 \mathrm{~min}$ ) & 2. LPF at $0.1 \mathrm{~Hz}$ \\
\hline & & 3. $7 x ; 45 \mathrm{~s}$ & 3. Averaging \\
\hline & & 4. 2 min after block & 4. Oxy- and deoxyHb \\
\hline & & & 5. Last $10 \mathrm{~s}$ of perturbation \\
\hline \multirow[t]{5}{*}{ Beurskens et al. ${ }^{105}$} & $\begin{array}{l}\text { - Healthy young adults } \\
\quad(n=15 ; 24.5 \pm 3.3)\end{array}$ & 1. Sitting on chair & 1. Constant value (6.0) \\
\hline & $\begin{array}{l}\text { - Healthy old adults } \\
\quad(n=10 ; 71.0 \pm 3.8)\end{array}$ & 2. $30 \mathrm{~s}$ & $\begin{array}{l}\text { 2. HRF-filter; wavelet MDL detrending } \\
\text { algorithm }\end{array}$ \\
\hline & \multirow[t]{3}{*}{ - DTW vs. NW } & 3. $2 \times ; 30 \mathrm{~s}$ & $\begin{array}{l}\text { 3. Moving standard deviation and } \\
\text { spline interpolation, baseline } \\
\text { correction, canonical HRF }\end{array}$ \\
\hline & & 4. Not reported & 4. Oxy- and deoxyHb \\
\hline & & & 5. Entire task time \\
\hline \multirow[t]{5}{*}{ Caliandro et al. ${ }^{70}$} & $\begin{array}{l}\text { - Patients with ataxic gait } \\
\quad(n=14 ; 27 \text { to } 71)\end{array}$ & 1. Quiet standing & 1. Constant value (5.93) \\
\hline & - Healthy controls $(n=20 ; 32$ to 65$)$ & 2. Last $10 \mathrm{~s}$ of standing & 2. LPF at $0.1 \mathrm{~Hz}$ \\
\hline & - Patients vs. HC & 3. $1 \times ; 10 \mathrm{~m}$ & 3. Baseline correction; averaging \\
\hline & & 4. Not relevant & 4. $\mathrm{OxyHb}$ \\
\hline & & & 5. Entire task time expect of first $5 \mathrm{~s}$ \\
\hline \multirow[t]{5}{*}{ Caliandro et al. ${ }^{71}$} & $\begin{array}{l}\text { - Patients with ataxic gait } \\
\quad(n=19 ; 31 \text { to } 70)\end{array}$ & 1. Quiet standing & 1. Constant value (5.93) \\
\hline & - Healthy controls $(n=15 ; 36$ to 73$)$ & 2. Last $10 \mathrm{~s}$ of standing & 2. LPF at $0.1 \mathrm{~Hz}$ \\
\hline & \multirow[t]{3}{*}{ - Patients vs. HC } & 3. $2 \times ; 10 \mathrm{~m}$ & 3. Baseline correction; averaging \\
\hline & & 4. 30 min between trials & 4. OxyHb \\
\hline & & & 5. Entire task time expect of first $5 \mathrm{~s}$ \\
\hline
\end{tabular}


Table 1 (Continued).

\begin{tabular}{|c|c|c|c|}
\hline \multirow[t]{5}{*}{ Clark et al. ${ }^{66}$} & $\begin{array}{l}\text { - Older persons with mobility and } \\
\text { somatosensory deficits } \\
(n=14 ; 77.1 \pm 5.56)\end{array}$ & 1. Quiet standing & 1. N/A \\
\hline & \multirow[t]{4}{*}{$\begin{array}{l}\text { - Walking in normal shoes vs. texture } \\
\text { insoles vs. barefoot vs. DTW }\end{array}$} & $\begin{array}{l}\text { 2. } 10 \mathrm{~s} \text { immediately before } \\
\text { task }\end{array}$ & 2. No filter \\
\hline & & $\begin{array}{l}\text { 3. } 5 \times \text { walking laps with } \\
18 \text { m (overground); } \\
60 \text { to } 120 \text { s (treadmill) }\end{array}$ & 3. Averaging \\
\hline & & 4. 2 min after task & 4. TOI \\
\hline & & & 5. Entire task phase \\
\hline \multirow[t]{5}{*}{ Clark et al. ${ }^{.8}$} & $\begin{array}{l}\text { - Older adults with mild mobility } \\
\text { deficits }(n=16 ; 77.2 \pm 5.6)\end{array}$ & 1. Quiet standing & 1. N/A \\
\hline & \multirow[t]{4}{*}{ - NW vs. DTW } & $\begin{array}{l}\text { 2. } 10 \mathrm{~s} \text { immediately before } \\
\text { task }\end{array}$ & 2. No filter \\
\hline & & 3. $5 \times$ walking laps with $18 \mathrm{~m}$ & 3. Averaging \\
\hline & & 4. 2 min after task & 4. TOI \\
\hline & & & $\begin{array}{l}\text { 5. } 10 \mathrm{~s} \text { before task begin (preparation } \\
\text { phase) and in steady phase/transition } \\
\text { phase excluded }\end{array}$ \\
\hline \multirow[t]{5}{*}{ Doi et al. ${ }^{72}$} & $\begin{array}{l}\text { - Adults with mild cognitive } \\
\text { impairment }(n=16 ; 75.4 \pm 7.2)\end{array}$ & 1. Quiet standing & 1. N/A (arbitrary unit) \\
\hline & \multirow[t]{4}{*}{ - NW vs. DTW } & 2. $10 \mathrm{~s}$ before walking & $\begin{array}{l}\text { 2. LPF at } 0.05 \mathrm{~Hz} \text {; linear fitting on } \\
\text { baseline data }\end{array}$ \\
\hline & & 3. $3 x ; 20 \mathrm{~s}$ & 3. Averaging \\
\hline & & 4. $30 \mathrm{~s}$ between trials & 4. ОхуHb \\
\hline & & & 5. Entire task period \\
\hline \multirow[t]{5}{*}{ Eggenberger et al. ${ }^{68}$} & $\begin{array}{l}\text { - Healthy old adults (dancing: } n=19 \\
72.8 \pm 5.9 ; \text { balance: } n=14 \\
77.8 \pm 7.4 \text { ) }\end{array}$ & 1. Walking at $2 \mathrm{~km} / \mathrm{h}$ & 1. N/A (absolute values) \\
\hline & \multirow[t]{4}{*}{$\begin{array}{l}\text { - Dancing vs. balancing } \\
\text { (before and after intervention) }\end{array}$} & 2. Middle $40 \mathrm{~s}$ (of $1 \mathrm{~min}$ ) & $\begin{array}{l}\text { 2. } 60 \mathrm{~s} \text { moving average: motion artifact } \\
\text { correction (oxyHb: }>2.5 \text { and } \\
<-2.5 \mu \mathrm{M} / \text { deoxyHb: }>1.5 \text { and } \\
<-1.5 \mu \mathrm{M} \text { excluded); visual } \\
\text { inspection of data }\end{array}$ \\
\hline & & 3. $8 x ; 30 \mathrm{~s}$ & 3. Averaging \\
\hline & & \multirow{2}{*}{$\begin{array}{l}\text { 4. } 30 \mathrm{~s} \text { between trials } \\
\text { (walking at } 2 \mathrm{~km} / \mathrm{h} \text { ) }\end{array}$} & 4. OxyHb \\
\hline & & & $\begin{array}{l}\text { 5. } 1 \text { to } 7 \mathrm{~s}=\text { acceleration phase; } \\
10 \text { to } 25 \mathrm{~s}=\text { steady state walking } \\
\text { phase; } 26 \text { to } 34 \mathrm{~s}=\text { deceleration } \\
\text { phase } 35 \text { to } 46 \mathrm{~s}=\text { drop phase }\end{array}$ \\
\hline \multirow[t]{5}{*}{ Ferrari et al. ${ }^{99}$} & $\begin{array}{l}\text { - Healthy, young adults } \\
\quad(n=22 ; 26.5 \pm 4.0)\end{array}$ & 1. Quiet standing & $\begin{array}{l}\text { 1. Age-dependent value } \\
\left(4.99+0.067 \times \text { age }^{0.814}\right)\end{array}$ \\
\hline & \multirow[t]{4}{*}{ - Balancing in semivirtual reality } & 2. Last $30 \mathrm{~s}$ (of $2 \mathrm{~min}$ ) & 2. LPF at $0.1 \mathrm{~Hz}$ \\
\hline & & 3. $2 x ; 9 \min$ & 3. Averaging \\
\hline & & 4. 2 min after block & 4. Oxy- and deoxyHb \\
\hline & & & 5. $30 \mathrm{~s}$ per task \\
\hline
\end{tabular}


Table 1 (Continued).

$$
\begin{array}{cc}
\text { Fraser et al. }^{63} & - \text { Healthy young adults } \\
& (n=19 ; 21.83 \pm 1.92) \\
- & \text { Healthy old adults } \\
& (n=14 ; 66.85 \pm 5.26)
\end{array}
$$

- NW vs. single cognitive task vs. easy DTW vs. hard DTW
1. Quiet standing

2. $5 \mathrm{~s}$

3. Walking: $2 x ; 2$ min; single cognitive task: $4 \times$; $75 \mathrm{~s}$; DTW 4x; 2 min (for each dual-task condition)

4. 30 to $60 \mathrm{~s}$ between trials
1. Constant value (no details reported)

\begin{tabular}{|c|c|c|c|}
\hline \multirow[t]{5}{*}{ Fujimoto et al. ${ }^{102}$} & $\begin{array}{l}\text { - Patients with subcortical stroke } \\
\quad(n=20 ; 60.2 \pm 9.5)\end{array}$ & 1. Quiet standing & 1. N/A (arbitrary unit) \\
\hline & \multirow[t]{4}{*}{$\begin{array}{l}\text { - Postural test before/after } \\
\text { rehabilitation }\end{array}$} & $\begin{array}{l}\text { 2. Time before next } \\
\text { perturbation (ERD) }\end{array}$ & 2. $\mathrm{HPF}$ at $0.01 \mathrm{~Hz} ; \mathrm{PCA}$ \\
\hline & & 3. $15 \mathrm{x} ; 1 \mathrm{~s}$ & 3. Two parameter gamma HRF \\
\hline & & \multirow{2}{*}{$\begin{array}{l}\text { 4. } 5 \text { to } 15 \mathrm{~s} \text { between trials } \\
\text { (randomized) }\end{array}$} & 4. Oxy- and deoxyHb \\
\hline & & & 5. Around perturbations \\
\hline \multirow[t]{5}{*}{ Fujita et al. ${ }^{101}$} & $\begin{array}{l}\text { - Healthy, young adults (low span } \\
\text { group: } n=13 ; 24.0 \pm 3.1 \text { / high } \\
\text { span group: } n=16 ; 22.5 \pm 3.6 \text { ) }\end{array}$ & 1. Quiet standing & 1. N/A (arbitrary unit) \\
\hline & \multirow[t]{4}{*}{$\begin{array}{l}\text { - Single- and dual-task mono- or } \\
\text { bipedal standing }\end{array}$} & 2. $10 \mathrm{~s}$ & $\begin{array}{l}\text { 2. LPF at } 0.5 \mathrm{~Hz} \text {; HPF at } 0.01 \mathrm{~Hz} \text {; } \\
5 \mathrm{~s} \text { moving average }\end{array}$ \\
\hline & & 3. $3 x ; 20 s$ & $\begin{array}{l}\text { 3. Baseline normalization, } \\
\text { baseline correction, averaging }\end{array}$ \\
\hline & & 4. $10 \mathrm{~s}$ between trials & 4. OxyHb \\
\hline & & & 5. Entire task time \\
\hline \multirow{5}{*}{ Harada et al. ${ }^{53}$} & \multirow{5}{*}{$\begin{array}{l}\text { - Healthy, old adults }(n=15 ; 63 \pm 4) \\
\text { - Low vs. high gait capacity group at } \\
\text { different speeds }\end{array}$} & 1. Quiet standing & 1. N/A (arbitrary unit) \\
\hline & & 2. $10 \mathrm{~s}$ before walking & 2. $\mathrm{HPF}$ at $0.03 \mathrm{~Hz}$ \\
\hline & & 3. $3 \times ; 60 \mathrm{~s}$ & 3. Baseline normalization; averaging \\
\hline & & 4. $40 \mathrm{~s}$ between trials & 4. $\mathrm{OxyHb}$ \\
\hline & & & 5. $20 \mathrm{~s}$ after target speed \\
\hline \multirow[t]{5}{*}{ Helmich et al. ${ }^{108}$} & $\begin{array}{l}\text { - Young, concussed adults with } \\
\text { persistent postconcussive } \\
\text { symptoms }(n=7 ; 29 \pm 15)\end{array}$ & 1. N/A & 1. Constant value (6.0) \\
\hline & $\begin{array}{l}\text { - Young, concussed adults with } \\
\text { minor postconcussive symptoms } \\
(n=13 ; 26 \pm 7)\end{array}$ & 2. N/A & $\begin{array}{l}\text { 2. LPF at } 0.1 \mathrm{~Hz} ; \mathrm{HPF} \text { at } 0.001 \mathrm{~Hz} \text {; } \\
\text { spline interpolation; visual inspection }\end{array}$ \\
\hline & - Healthy, young adults $(n=10 ; 27 \pm 8)$ & 3. $10 x ; 10 s$ & 3. Normalization; averaging \\
\hline & \multirow{2}{*}{$\begin{array}{l}\text { - Comparison of three groups during } \\
\text { standing on different surfaces } \\
\text { (stable vs. instable) and sensory } \\
\text { conditions (eyes closed vs. eyes } \\
\text { open vs. blurred vision) }\end{array}$} & \multirow[t]{2}{*}{ 4. No rest between trials } & 4. Oxy- and deoxyHb \\
\hline & & & 5. Entire task time \\
\hline \multirow[t]{5}{*}{ Hernandez et al. ${ }^{82}$} & - Healthy old adults $(n=8 ; 61 \pm 4)$ & 1. Quiet standing & 1. Constant value (6.0) \\
\hline & $\begin{array}{l}\text { - Patients with multiple sclerosis } \\
\quad(n=8 ; 57 \pm 5)\end{array}$ & $\begin{array}{l}\text { 2. } 10 \mathrm{~s} \text { before walking } \\
\text { (counting silently in } \\
\text { steps of } 1 \text { ) }\end{array}$ & $\begin{array}{l}\text { 2. LPF at } 0.14 \mathrm{~Hz} \text {; noisy channels } \\
\text { excluded (dark current condition or } \\
\text { saturation); visual inspected }\end{array}$ \\
\hline & \multirow{3}{*}{$\begin{array}{l}\text { - Comparison of healthy adults and } \\
\text { patients with multiple sclerosis during } \\
\text { NW and DTW }\end{array}$} & $\begin{array}{l}\text { 3. } 3 \times \text { walking loops }(=6 \times \\
\text { straight walks a } 14 \mathrm{ft} .)\end{array}$ & 3. Baseline normalization; averaging \\
\hline & & \multirow[t]{2}{*}{ 4. At least $10 \mathrm{~s}$ after trial } & 4. Охунb \\
\hline & & & 5. Entire task time \\
\hline
\end{tabular}

2. No filter

3. Averaging

4. Oxy- and deoxyHb

5. Entire task period 
Table 1 (Continued).

\begin{tabular}{|c|c|c|c|}
\hline \multirow[t]{5}{*}{ Herold et al. ${ }^{100}$} & $\begin{array}{l}\text { - Healthy young adults } \\
\quad(n=10 ; 25 ; 21 \text { to } 47)\end{array}$ & 1. Quiet standing & 1. N/A (arbitrary unit) \\
\hline & \multirow[t]{4}{*}{$\begin{array}{l}\text { - Standing vs. balancing on } \\
\text { balance board }\end{array}$} & 2. $30 \mathrm{~s}$ before task & $\begin{array}{l}\text { 2. } 5.0 \text { s moving average; LPF at } 0.5 \mathrm{~Hz} \text {; } \\
\mathrm{HPF} \text { at } 0.01 \mathrm{~Hz} \text {; PCA }(r=0.25)\end{array}$ \\
\hline & & 3. $3 x ; 30 \mathrm{~s}$ & 3. Averaging \\
\hline & & 4. $30 \mathrm{~s}$ after trial & 4. Oxy- and deoxyHb \\
\hline & & & 5. Middle $20 \mathrm{~s}$ \\
\hline \multirow[t]{5}{*}{ Holtzer et al. ${ }^{73}$} & - Healthy, young adults ( $n=11 ; 19$ to 29 ) & 1. Quiet standing & 1. Constant value $(6.0)$ \\
\hline & - Healthy, old adults ( $n=11 ; 69$ to 88 ) & 2. $5 \mathrm{~s}$ before walking & $\begin{array}{l}\text { 2. LPF at } 0.14 \mathrm{~Hz} \text {; combined principal and } \\
\text { independent component analysis }\end{array}$ \\
\hline & \multirow[t]{3}{*}{$\begin{array}{l}\text { - DTW vs. NW vs. standing/comparison } \\
\text { between cohorts }\end{array}$} & $\begin{array}{l}\text { 3. } 3 \times \text { walking loops }(=6 \times \\
\text { straight walks a } 15 \mathrm{ft} .)\end{array}$ & 3. Baseline normalization; averaging \\
\hline & & 4. Not reported & 4. ОxyHb \\
\hline & & & 5. Old $4 \mathrm{~s} /$ young $3.5 \mathrm{~s}$ \\
\hline \multirow[t]{5}{*}{ Holtzer et al. ${ }^{74}$} & $\begin{array}{l}\text { - Nondemented older adults } \\
\quad(n=318 ; 76.66 \pm 6.7)\end{array}$ & 1. Quiet standing & 1. Constant value (6.0) \\
\hline & \multirow[t]{4}{*}{ - DTW vs. NW vs. standing } & $\begin{array}{l}\text { 2. } 10 \mathrm{~s} \text { (counting silently } \\
\text { forward in steps of } 1 \text { ) }\end{array}$ & $\begin{array}{l}\text { 2. LPF at } 0.14 \mathrm{~Hz} \text {; noisy channels } \\
\text { excluded (dark current condition or } \\
\text { saturation); visual inspected }\end{array}$ \\
\hline & & $\begin{array}{l}\text { 3. } 3 \times \text { walking loops }(=6 \times \\
\text { straight walks a } 14 \mathrm{ft} .) / \\
\text { standing for } 30 \mathrm{~s}\end{array}$ & 3. Baseline normalization; averaging \\
\hline & & 4. "Short break" reported & 4. OxyHb \\
\hline & & & 5. Entire task time \\
\hline \multirow[t]{5}{*}{ Holtzer et al. ${ }^{84}$} & $\begin{array}{l}\text { - Nondemented older adults } \\
\quad(n=348 ; 76.8 \pm 6.8)\end{array}$ & 1. Quiet standing & 1. Constant value (6.0) \\
\hline & $\begin{array}{l}\text { - Older adults with low perceived stress } \\
\quad(n=147 ; 76.72 \pm 6.87)\end{array}$ & $\begin{array}{l}\text { 2. } 10 \mathrm{~s} \text { (counting silently } \\
\text { forward in steps of } 1 \text { ) }\end{array}$ & $\begin{array}{l}\text { 2. LPF at } 0.14 \mathrm{~Hz} \text {; noisy channels } \\
\text { excluded (dark current condition or } \\
\text { saturation); visual inspected }\end{array}$ \\
\hline & $\begin{array}{l}\text { - Older adults high perceived stress } \\
\quad(n=171 ; 76.58 \pm 6.37)\end{array}$ & $\begin{array}{l}\text { 3. } 3 \times \text { walking loops }(=6 \times \\
\text { straight walks a } 14 \mathrm{ft} .) / \\
\text { standing for } 30 \mathrm{~s}\end{array}$ & 3. Baseline normalization; averaging \\
\hline & \multirow{2}{*}{$\begin{array}{l}\text { - DTW vs. NW vs. standing/comparison } \\
\text { between cohorts }\end{array}$} & \multirow[t]{2}{*}{ 4. "Short break" reported } & 4. $\mathrm{OxyHb}$ \\
\hline & & & 5. Entire task time \\
\hline \multirow[t]{5}{*}{ Holtzer et al. ${ }^{75}$} & $\begin{array}{l}\text { - Nondemented older adults } \\
\text { (total: } n=236 ; 75.5 \pm 6.49 \text { ) }\end{array}$ & 1. Quiet standing & 1. Constant value (6.0) \\
\hline & $\begin{array}{l}\text { - Healthy older adults } \\
\qquad(n=167 ; 74.43 \pm 6.04)\end{array}$ & $\begin{array}{l}\text { 2. } 10 \mathrm{~s} \text { (counting silently } \\
\text { forward in steps of } 1 \text { ) }\end{array}$ & $\begin{array}{l}\text { 2. LPF at } 0.14 \mathrm{~Hz} \text {; noisy channels } \\
\text { excluded (dark current condition or } \\
\text { saturation); visual inspected }\end{array}$ \\
\hline & $\begin{array}{l}\text { - Older adults with peripheral NGA } \\
\quad(n=40 ; 77.03 \pm 6.27)\end{array}$ & $\begin{array}{l}\text { 3. } 3 \times \text { walking loops }(=6 \times \\
\text { straight walks a } 14 \mathrm{ft} .) / \\
\text { standing for } 30 \mathrm{~s}\end{array}$ & 3. Baseline normalization; averaging \\
\hline & $\begin{array}{l}\text { - Older adults with central NGA } \\
\quad(n=29 ; 79.59 \pm 7.38)\end{array}$ & 4. "Short break" reported & 4. OxyHb \\
\hline & \multicolumn{2}{|l|}{$\begin{array}{l}\text { - DTW vs. NW vs. standing/comparison } \\
\text { between cohorts }\end{array}$} & 5. Entire task time \\
\hline
\end{tabular}


Table 1 (Continued).

\begin{tabular}{|c|c|c|c|}
\hline \multirow[t]{5}{*}{ Holtzer et al. ${ }^{85}$} & $\begin{array}{l}\text { - Older adults with low perceived } \\
\text { fatigue }(n=160 ; 76.20 \pm 6.64)\end{array}$ & 1. Quiet standing & 1. Constant value $(6.0)$ \\
\hline & $\begin{array}{l}\text { - Older adults with high perceived fatigue } \\
\qquad(n=154 ; 77.41 \pm 6.66)\end{array}$ & $\begin{array}{l}\text { 2. } 10 \mathrm{~s} \text { (counting silently } \\
\text { forward in steps of } 1 \text { ) }\end{array}$ & $\begin{array}{l}\text { 2. LPF at } 0.14 \mathrm{~Hz} \text {; noisy channels } \\
\text { excluded (dark current condition or } \\
\text { saturation); visual inspected }\end{array}$ \\
\hline & $\begin{array}{l}\text { - DTW vs. NW vs. standing/comparison } \\
\text { between cohorts }\end{array}$ & $\begin{array}{l}\text { 3. } 3 \times \text { walking loops }(=6 \times \\
\text { straight walks a } 14 \mathrm{ft} .) / \\
\text { standing for } 30 \mathrm{~s}\end{array}$ & 3. Baseline normalization; averaging \\
\hline & & 4. "Short break" reported & 4. OxyHb \\
\hline & & & 5. Entire task time \\
\hline \multirow[t]{5}{*}{ Huppert et al. ${ }^{91}$} & - Healthy young adults ( $n=10 ; 21$ to 47 ) & 1. Quiet standing & 1. Not relevant (image reconstruction) \\
\hline & - Stepping reaction task & $\begin{array}{l}\text { 2. Time before next trial } \\
\text { ( } 4 \text { to } 8 \mathrm{~s} \text {, random order) }\end{array}$ & $\begin{array}{l}\text { 2. Discrete cosinus transform term } \\
(0-1 / 120 \mathrm{~Hz}) \text {; visual inspected }\end{array}$ \\
\hline & & 3. $8 \times$ blocks a $32 \times$ trials & 3. Gamma-variant HRF; averaging \\
\hline & & 4. 4 to $8 \mathrm{~s}$ between trials & 4. Oxy- and deoxyHb \\
\hline & & $\begin{array}{l}\text { (random order) / few } \\
\text { minutes after } 2 \text { to } 3 \text { scans }\end{array}$ & 5. Entire task phase \\
\hline \multirow[t]{5}{*}{ Karim et al. ${ }^{97}$} & - Healthy young adults ( $n=9 ; 18$ to 42 ) & 1. Quiet standing & 1. Not relevant (image reconstruction) \\
\hline & - Video game with balance task & 2. $60 \mathrm{~s}$ (pre- and posttask) & $\begin{array}{l}\text { 2. Cosinus transform term } \\
(0 \text { to } 1 / 120 \mathrm{~Hz}) \text {; visual inspected }\end{array}$ \\
\hline & & $\begin{array}{l}\text { 3. } 6 \times \text { beginner } / 8 \times \text { advanced } \\
\text { level; } 30 \text { to } 60 \mathrm{~s}\end{array}$ & 3. Boxcar HRF; averaging \\
\hline & & 4. $30 \mathrm{~s}$ between trials & 4. Oxy- and deoxyHb \\
\hline & & & 5. Entire task phase \\
\hline \multirow[t]{5}{*}{ Karim et al. ${ }^{92}$} & $\begin{array}{l}\text { - Healthy young adults } \\
\quad(n=15 ; 28 \pm 9)\end{array}$ & 1. Quiet standing & 1. Not relevant (image reconstruction) \\
\hline & - SOT conditions & 2. $45 \mathrm{~s}$ before trial & $\begin{array}{l}\text { 2. Cosinus transform term } \\
\text { (0 to } 1 / 120 \mathrm{~Hz})\end{array}$ \\
\hline & & 3. $2 x ; 45 \mathrm{~s}$ & 3. Gamma-variant HRF; averaging \\
\hline & & 4. $60 \mathrm{~s}$ after trial / 2 min after & 4. Oxy- and deoxyHb \\
\hline & & two scans & 5. Entire task phase \\
\hline \multirow[t]{5}{*}{ Kim et al. ${ }^{106}$} & $\begin{array}{l}\text { - Healthy young adults } \\
\quad(n=14 ; 30.06 \pm 4.53)\end{array}$ & 1. Not reported & 1. Not reported \\
\hline & $\begin{array}{l}\text { - Stepping (ST) vs. Treadmill walking } \\
\text { (TW) vs. robot-assisted walking (RAW) }\end{array}$ & 2. Not reported & $\begin{array}{l}\text { 2. Gaussian smoothing; wavelet MDL } \\
\text { algorithm }\end{array}$ \\
\hline & & $\begin{array}{l}\text { 3. } 5 \times ; 30 \mathrm{~s}(\mathrm{ST}, \mathrm{TW}) \\
60 \mathrm{~s} \text { (RAW) }\end{array}$ & 3. Canonical HRF \\
\hline & & 4. $15 \mathrm{~s}$ at begin and end; $30 \mathrm{~s}$ & 4. OxyHb \\
\hline & & $\begin{array}{l}\text { between trials (ST, TW) / } \\
60 \mathrm{~s} \text { at begin and end; } \\
45 \mathrm{~s} \text { between trials (RAW) }\end{array}$ & 5. Entire task time \\
\hline \multirow[t]{5}{*}{ Koenraadt et al. ${ }^{54}$} & - Healthy, young adults $(n=11 / 23 \pm 4)$ & 1. Quiet standing & 1. N/A (arbitrary unit) \\
\hline & - Precision walking vs. NW & 2. 25 to $35 \mathrm{~s}$ & $\begin{array}{l}\text { 2. LPF at } 1.25 \mathrm{~Hz} \text {; HPF at } 0.01 \mathrm{~Hz} \text {; } \\
\text { superficial interference with LPF at } \\
1 \mathrm{~Hz} \text {; short separation channels }(1 \mathrm{~cm})\end{array}$ \\
\hline & & 3. $10 x ; 35 \mathrm{~s}$ & 3. Baseline normalization; averaging \\
\hline & & 4. 25 to $35 \mathrm{~s}$ before/after & 4. Oxy- and deoxyHb \\
\hline & & & 5. $12.5 \mathrm{~s}$ in task phase \\
\hline
\end{tabular}


Table 1 (Continued).

\begin{tabular}{|c|c|c|c|}
\hline \multirow[t]{5}{*}{ Kurz et al. ${ }^{55}$} & $\begin{array}{l}\text { - Healthy, young adults } \\
\quad(n=13 ; 23.7 \pm 1.4)\end{array}$ & 1. Quiet standing & 1. N/A (arbitrary unit) \\
\hline & \multirow[t]{4}{*}{ - Forward vs. backward walking } & 2. $2.5 \mathrm{~s}$ before walking & $\begin{array}{l}\text { 2. HPF at } 0.01 \mathrm{~Hz} ; 5 \text { s moving average; } \\
\text { PCA }(r=0.25)\end{array}$ \\
\hline & & 3. $10 x ; 30 \mathrm{~s}$ & 3. Baseline correction; averaging \\
\hline & & 4. $30 \mathrm{~s}$ between trials & 4. Oxy- and deoxyHb \\
\hline & & & 5. Entire task phase \\
\hline \multirow[t]{5}{*}{ Kurz et al. ${ }^{56}$} & $\begin{array}{l}\text { - Children with spastic diplegic cerebral } \\
\text { palsy }(n=4 ; 11.0 \pm 4)\end{array}$ & 1. Quiet standing & 1. N/A (arbitrary unit) \\
\hline & - Healthy children $(n=8 ; 13.2 \pm 3)$ & 2. $2.5 \mathrm{~s}$ before walking & $\begin{array}{l}\text { 2. HPF at } 0.01 \mathrm{~Hz} ; 5 \text { s moving average; } \\
\text { PCA }(r=0.25)\end{array}$ \\
\hline & \multirow[t]{3}{*}{ - Patients vs. HC } & 3. $10 \times ; 30 \mathrm{~s}$ & 3. Baseline correction; averaging \\
\hline & & 4. $30 \mathrm{~s}$ between trials & 4. $\mathrm{OxyHb}$ \\
\hline & & & 5. Entire task phase \\
\hline \multirow[t]{5}{*}{ Lin et al. ${ }^{103}$} & $\begin{array}{l}\text { - Healthy middle-aged adults } \\
\quad(n=15 ; 46 \pm 11)\end{array}$ & 1. Quiet standing & 1. N/A (image reconstruction) \\
\hline & - Healthy old adults $(n=15 ; 73 \pm 5)$ & 2. $40 \mathrm{~s}$ before trial & $\begin{array}{l}\text { 2. Autoregressive model with } \\
\text { prewhitened iterative reweighted } \\
\text { least squares algorithm }\end{array}$ \\
\hline & \multirow{3}{*}{$\begin{array}{l}\text { - Middle-aged vs. old adults } \\
\text { (different balance conditions) }\end{array}$} & 3. $4 \times ; 40 \mathrm{~s}$ & 3. HRF; averaging \\
\hline & & 4. $1 \mathrm{~min}$ between trials & 4. Oxy- and deoxyHb \\
\hline & & & 5. Entire task phase \\
\hline \multirow[t]{5}{*}{ Lin and $\operatorname{Lin}^{79}$} & - Healthy young adults ( $n=24 ; 20$ to 27 ) & 1. Quiet standing & $\begin{array}{l}\text { 1. Age-dependent value } \\
\left(4.99+0.067 \times \text { age }^{0.814}\right)\end{array}$ \\
\hline & \multirow[t]{4}{*}{ - DTW vs. NW } & 2. $20 \mathrm{~s}$ & 2. LPF at $0.2 \mathrm{~Hz}$ \\
\hline & & 3. $1 \times ; 60 \mathrm{~s}$ & 3. Baseline correction \\
\hline & & 4. $20 \mathrm{~s}$ before/after task / & 4. ОxyHb \\
\hline & & 2 min after two trials & 5. Entire task phase \\
\hline \multirow[t]{5}{*}{ Lu et al. ${ }^{76}$} & $\begin{array}{l}\text { - Healthy young adults } \\
\quad(n=17 ; 23.1 \pm 1.5)\end{array}$ & 1. Quiet standing & 1. Constant value (6.0) \\
\hline & \multirow[t]{4}{*}{ - DTW vs. NW } & 2. $5 \mathrm{~s}$ before walking & $\begin{array}{l}\text { 2. Bandpass filter (LPF at } 0.01 \mathrm{~Hz} \text {; HPF at } \\
0.2 \mathrm{~Hz} \text { ); PCA; spike rejection (channels } \\
\text { with }>\mathrm{CV} 15 \% \text { rejected/channels with } \\
\mathrm{CV}>10 \% \text { for further analysis) }\end{array}$ \\
\hline & & 3. $3 \times ; 60 \mathrm{~s}$ & 3. Averaging \\
\hline & & 4. $60 \mathrm{~s}$ between trials & 4. Hbdiff (oxyHb-deoxyHb) \\
\hline & & & $\begin{array}{l}\text { 5. Early phase ( } 5 \text { to } 20 \mathrm{~s} \text { after task begin); } \\
\text { late phase ( } 21 \text { to } 50 \mathrm{~s} \text { after task begin) }\end{array}$ \\
\hline \multirow[t]{5}{*}{ Mahoney et al. ${ }^{93}$} & $\begin{array}{l}\text { - Healthy, nondemented older adults } \\
\quad(n=126 ; 74.41 \pm 6.12)\end{array}$ & 1. Quiet standing & 1. Constant value $(6.0)$ \\
\hline & $\begin{array}{l}\text { - Older adults wild mild Parkinson } \\
\text { symptoms }(n=117 ; 77.50 \pm 6.72)\end{array}$ & 2. First $2 \mathrm{~s}$ & 2. LPF at $0.14 \mathrm{~Hz}$; visual inspected \\
\hline & $\begin{array}{l}\text { - Patients with Parkinson disease } \\
\quad(n=26 ; 81.23 \pm 5.93)\end{array}$ & 3. $10 \mathrm{~s}$ & 3. Baseline normalization; averaging \\
\hline & \multirow{2}{*}{$\begin{array}{l}\text { - Patients vs. HC (standing while } \\
\text { counting silently in steps of 1) }\end{array}$} & \multirow[t]{2}{*}{ 4. "Short break" reported } & 4. OxyHb \\
\hline & & & 5. Entire task phase \\
\hline
\end{tabular}


Table 1 (Continued).

\begin{tabular}{|c|c|c|c|}
\hline \multirow[t]{5}{*}{ Maidan et al. ${ }^{90}$} & $\begin{array}{l}\text { - Parkinson patients with FOG } \\
\quad(n=11 ; 66.2 \pm 10.0)\end{array}$ & 1. Walking & $\begin{array}{l}\text { 1. Age-dependent value } \\
\left(4.99+0.067 \times \text { age }^{0.814}\right)\end{array}$ \\
\hline & - Healthy controls $(n=11 ; 71.2 \pm 6.0)$ & 2. $6 \mathrm{~s}$ before $\mathrm{FOG}$ & 2. LPF at $0.14 \mathrm{~Hz}$ \\
\hline & - Patients vs. HC (walking; turning) & $\begin{array}{l}\text { 3. } 6 \mathrm{~s} \text { walking with } 180 \mathrm{deg} \\
\text { turn }\end{array}$ & 3. Baseline correction; averaging \\
\hline & & 4. 2 min between tasks & 4. OxyHb \\
\hline & & & $\begin{array}{l}\text { 5. Defined time period around FOG event } \\
\text { (prior }=-6 \text { to }-3 \mathrm{~s} / \text { before }=-3 \text { to } \\
0 / \text { during }=0 \text { to } 3 \mathrm{~s} \text { ) }\end{array}$ \\
\hline \multirow[t]{5}{*}{ Maidan et al. ${ }^{80}$} & $\begin{array}{l}\text { - Healthy, older adults } \\
\quad(n=38 ; 70.4 \pm 0.9)\end{array}$ & 1. Quiet standing & $\begin{array}{l}\text { 1. Age-dependent value } \\
\left(4.99+0.067 \times \text { age }^{0.814}\right)\end{array}$ \\
\hline & - Parkinson patients $(n=68 ; 71.7 \pm 1.1)$ & 2. $5 \mathrm{~s}$ before task & $\begin{array}{l}\text { 2. Bandpass filter (LPF at } 0.01 \mathrm{~Hz} \text { and } \\
\text { HPF at } 0.14 \mathrm{~Hz} \text { ), wavelet filter; CBSI }\end{array}$ \\
\hline & - DTW vs. NW vs. obstacle negotiation & 3. $5 x ; 30 s$ & 3. Baseline correction; averaging \\
\hline & & 4. $20 \mathrm{~s}$ after trial / between & 4. OxyHb \\
\hline & & trials on individual needs & 5. Entire task phase \\
\hline \multirow[t]{5}{*}{ McKendrick et al. ${ }^{88}$} & $\begin{array}{l}\text { - Healthy, young adults } \\
(n=13 ; 22 ; 19 \text { to } 31)\end{array}$ & $\begin{array}{l}\text { 1. Sitting (for sitting condition) } \\
\text { and standing (for walking } \\
\text { condition) }\end{array}$ & 1. Constant value (5.94) \\
\hline & - Sitting vs. walking indoors vs. walking & 2. $10 \mathrm{~s}$ & 2. LPF at $0.1 \mathrm{~Hz}$; visual inspected \\
\hline & $\begin{array}{l}\text { outdoors (all conditions while } \\
\text { performing secondary task) }\end{array}$ & $\begin{array}{l}\text { 3. } 16 \times ; 120 \text { s (sitting) / } 8 \times \text {; } \\
\text { a } 120 \mathrm{~s} \text { (per walking } \\
\text { condition) }\end{array}$ & 3. Baseline correction \\
\hline & & 4. 5 min between walking & 4. Oxy- and deoxyHb \\
\hline & & conditıons & 5. Entire task time \\
\hline \multirow[t]{5}{*}{ Meester et al. ${ }^{57}$} & $\begin{array}{l}\text { - Young, healthy adults } \\
\quad(n=17 ; 27.8 \pm 6.3)\end{array}$ & 1. Quiet standing & $\begin{array}{l}\text { 1. Age-dependent value } \\
\left(4.99+0.067 \times \text { age }^{0.814}\right)\end{array}$ \\
\hline & - DTW vs. NW & 2. Middle $10 \mathrm{~s}$ of rest & $\begin{array}{l}\text { 2. LPF at } 0.67 \mathrm{~Hz} \text {; } 4 \mathrm{~s} \text { moving average; } \\
\text { visual inspected }\end{array}$ \\
\hline & & 3. $5 \times ; 30 \mathrm{~s}$ & 3. Averaging \\
\hline & & 4. 20 to $40 \mathrm{~s}$ between trials & 4. ОхуHb \\
\hline & & & 5. Middle $10 \mathrm{~s}$ of task \\
\hline \multirow[t]{5}{*}{ Metzger et al. ${ }^{64}$} & $\begin{array}{l}\text { - Healthy young adults } \\
\qquad(n=12 ; 27.6 ; 19 \text { to } 39)\end{array}$ & 1. Quiet standing & 1. N/A (arbitrary unit) \\
\hline & - DTW vs. NW & 2. $10 \mathrm{~s}$ at begin & 2. 5 s moving average; CBSI \\
\hline & & 3. $4 \times ; 45 \mathrm{~s}$ & 3. Averaging; baseline correction \\
\hline & & 4. $15 \mathrm{~s}$ after trial & 4. Oxy- and deoxyHb \\
\hline & & & 5. Entire task time \\
\hline \multirow[t]{5}{*}{ Mihara et al. ${ }^{58}$} & $\begin{array}{r}\text { - Stroke patients with ataxic gait } \\
(n=12 ; 52.7 \pm 16.9,12 \text { to } 74)\end{array}$ & 1. Quiet standing & 1. N/A (arbitrary unit) \\
\hline & $\begin{array}{l}\text { - Healthy controls } \\
\quad(n=11 ; 42.6 \pm 11.6,30 \text { to } 70)\end{array}$ & 2. $6 \mathrm{~s}$ before walking & 2. Not reported \\
\hline & - Patients vs. HC & $\begin{array}{l}\text { 3. } 3 \times ; 60 \mathrm{~s}(\mathrm{HC}) \\
30 \mathrm{~s} \text { (patients) }\end{array}$ & 3. Baseline correction; averaging \\
\hline & & 4. $15 \mathrm{~s}$ before/after walking & 4. $\mathrm{OxyHb}$ \\
\hline & & & $\begin{array}{l}\text { 5. Acceleration phase }=6 \mathrm{~s} \text { after starting } \\
\text { treadmill; steady phase }=6 \mathrm{~s} \text { during } \\
\text { steady speed }\end{array}$ \\
\hline
\end{tabular}


Table 1 (Continued).

\begin{tabular}{|c|c|c|c|}
\hline \multirow[t]{5}{*}{ Mihara et al. ${ }^{94}$} & $\begin{array}{l}\text { - Healthy young adults } \\
\quad(n=15 ; 29.4 \pm 6.7)\end{array}$ & 1. Quiet standing & 1. N/A (arbitrary unit) \\
\hline & \multirow{4}{*}{$\begin{array}{l}\text { - Warned before perturbations vs. } \\
\text { baseline; unwarned before } \\
\text { perturbations vs. baseline; } \\
\text { warned vs. unwarned }\end{array}$} & $\begin{array}{l}\text { 2. Time before next } \\
\text { perturbation (ERD) }\end{array}$ & 2. HPF at $0.05 \mathrm{~Hz}$ \\
\hline & & 3. 20 to $30 x ; 1 \mathrm{~s}$ & 3. Gaussian HRF; averaging \\
\hline & & \multirow{2}{*}{$\begin{array}{l}\text { 4. } 5 \text { to } 20 \mathrm{~s} \text { between trials } \\
\text { (randomized) / } 4 \text { to } 5 \mathrm{~min} \\
\text { after block }\end{array}$} & 4. OxyHb \\
\hline & & & 5. Around perturbation \\
\hline \multirow[t]{5}{*}{ Mihara et al. ${ }^{95}$} & \multirow{5}{*}{$\begin{array}{l}\text { - Stroke patients }(n=20 ; 61.6 \pm 11.9) \\
\text { - Balance perturbations }\end{array}$} & 1. Quiet standing & 1. N/A (arbitrary unit) \\
\hline & & $\begin{array}{l}\text { 2. Time before next } \\
\text { perturbation (ERD) }\end{array}$ & 2. $\mathrm{HPF}$ at $0.03 \mathrm{~Hz}$ \\
\hline & & 3. $15 \mathrm{x} ; 1 \mathrm{~s}$ & 3. Two-parameter gamma HRF \\
\hline & & 4. 5 to $15 \mathrm{~s}$ between trials & 4. OxyHb \\
\hline & & (randomized) & 5. Around perturbations \\
\hline \multirow[t]{5}{*}{ Mirelman et al. ${ }^{77}$} & $\begin{array}{l}\text { - Young, healthy adults } \\
\quad(n=23 ; 30.9 \pm 3.7)\end{array}$ & 1. Quiet standing & $\begin{array}{l}\text { 1. Age-dependent value } \\
\left(4.99+0.067 \times \text { age }^{0.814}\right)\end{array}$ \\
\hline & \multirow[t]{4}{*}{ - Standing vs. DTS vs. NW vs. DTW } & 2. $20 \mathrm{~s}$ before walking & $\begin{array}{l}\text { 2. LPF at } 0.14 \mathrm{~Hz} \text {; continuous wavelet } \\
\text { transform }\end{array}$ \\
\hline & & 3. $5 \times ; 30 \mathrm{~m}$ & 3. Baseline correction; averaging \\
\hline & & 4. $20 \mathrm{~s}$ before/after trial & 4. OxyHb \\
\hline & & & 5. Entire task phase \\
\hline \multirow[t]{5}{*}{ Miyai et al. ${ }^{107}$} & $\begin{array}{l}\text { - Healthy young adults } \\
\quad(n=8 ; 35 \pm 8,24 \text { to } 56)\end{array}$ & 1. Quiet standing & 1. N/A (arbitrary unit) \\
\hline & \multirow[t]{4}{*}{ - NW vs. standing } & 2. $30 \mathrm{~s}$ & 2. $\mathrm{HPF}$ at $0.03 \mathrm{~Hz}$ \\
\hline & & 3. $5 \mathrm{x} ; 30 \mathrm{~s}$ & 3. Linear interpolation; averaging \\
\hline & & 4. $30 \mathrm{~s}$ between trials & 4. Oxy- and deoxyHb \\
\hline & & & 5. Entire task phase \\
\hline \multirow[t]{5}{*}{ Miyai et al. ${ }^{61}$} & \multirow{5}{*}{$\begin{array}{l}\text { - Stroke patients }(n=6 ; 57 \pm 13) \\
\text { - Walking with mechanical assistance vs. } \\
\text { walking with facilitation technique }\end{array}$} & 1. Quiet standing & 1. N/A (arbitrary unit) \\
\hline & & 2. Middle $20 \mathrm{~s}$ & 2. HPF at $0.03 \mathrm{~Hz}$ \\
\hline & & 3. $4 \times ; 30 \mathrm{~s}$ & $\begin{array}{l}\text { 3. Linear interpolation; baseline } \\
\text { correction; averaging }\end{array}$ \\
\hline & & 4. $30 \mathrm{~s}$ between trials & 4. $\mathrm{OxyHb}$ \\
\hline & & & 5. Last $20 \mathrm{~s}$ of task phase \\
\hline \multirow[t]{5}{*}{ Miyai et al. ${ }^{60}$} & \multirow{5}{*}{$\begin{array}{l}\text { - Stroke patients }(n=8 ; 57 \pm 12) \\
\text { - Before/after } 2 \text { months rehabilitation }\end{array}$} & 1. Quiet standing & 1. N/A (arbitrary unit) \\
\hline & & 2. Middle $20 \mathrm{~s}$ & 2. HPF at $0.03 \mathrm{~Hz}$ \\
\hline & & 3. $4 \times ; 30 \mathrm{~s}$ & $\begin{array}{l}\text { 3. Linear interpolation; baseline } \\
\text { correction; averaging }\end{array}$ \\
\hline & & 4. $30 \mathrm{~s}$ between trials & 4. $\mathrm{OxyHb}$ \\
\hline & & & 5. Last $20 \mathrm{~s}$ of task phase \\
\hline \multirow[t]{5}{*}{ Miyai et al. ${ }^{59}$} & $\begin{array}{l}\text { - Stroke patients with hemiparesis } \\
(n=6 ; 57 \pm 6)\end{array}$ & 1. Quiet standing & 1. N/A (arbitrary unit) \\
\hline & - Healthy controls $(n=6,53 \pm 11)$ & 2. Middle $20 \mathrm{~s}$ & 2. $\mathrm{HPF}$ at $0.03 \mathrm{~Hz}$ \\
\hline & \multirow[t]{3}{*}{$\begin{array}{l}\text { - Walking with weight support vs. walking } \\
\text { without weight support }\end{array}$} & 3. $4 \times ; 30 \mathrm{~s}$ & $\begin{array}{l}\text { 3. Linear interpolation; baseline } \\
\text { correction; averaging }\end{array}$ \\
\hline & & 4. $30 \mathrm{~s}$ between trials & 4. ОхуHb \\
\hline & & & 5. Last $20 \mathrm{~s}$ of task phase \\
\hline
\end{tabular}


Table 1 (Continued).

\begin{tabular}{|c|c|c|c|}
\hline Nieuwhof et al. ${ }^{81}$ & $\begin{array}{l}\text { - Parkinson patients }(n=14 ; 71.2 \pm 5.4 \text { ) } \\
\text { - DTW (with different tasks) }\end{array}$ & $\begin{array}{l}\text { 1. Quiet standing } \\
\text { 2. Last } 5 \mathrm{~s} \text { of standing } \\
\text { 3. } 5 \times ; 40 \mathrm{~s} \\
\text { 4. } 20 \mathrm{~s} \text { between trials / } 1 \text { to } \\
2 \text { min between blocks }\end{array}$ & $\begin{array}{l}\text { 1. Constant value (6.0) } \\
\text { 2. LPF at } 0.1 \mathrm{~Hz} \text {; visual inspected } \\
\text { 3. Baseline correction; averaging } \\
\text { 4. OxyHb and deoxyHb } \\
\text { 5. Entire task phase }\end{array}$ \\
\hline Osofundiya et al. ${ }^{87}$ & $\begin{array}{l}\text { - Obese old adults }(n=10 ; 80.6 \pm 6.79) \\
\text { - nonobese old adults }(n=10 \\
80.6 \pm 7.50) \\
\text { - Quiet sitting vs. NW vs. precision } \\
\text { walking vs. DTW }\end{array}$ & $\begin{array}{l}\text { 1. Quiet standing } \\
\text { 2. } 10 \mathrm{~s} \\
\text { 3. } 8 \times \text { a } 30 \mathrm{~s} \\
\text { 4. } 10 \mathrm{~s} \text { between trials }\end{array}$ & $\begin{array}{l}\text { 1. Constant value (6.0) } \\
\text { 2. Not reported } \\
\text { 3. Baseline correction; averaging } \\
\text { 4. OxyHb and } \mathrm{HbT} \\
\text { 5. Entire task phase }\end{array}$ \\
\hline Saitou et al. ${ }^{78}$ & $\begin{array}{l}\text { - Hemiplegic stroke patients } \\
(n=44 ; 66 \pm 9.3) \\
\text { - Different tasks (e.g., calculation, pulley, } \\
\text { we only consider walking vs. baseline) }\end{array}$ & $\begin{array}{l}\text { 2. } 5 \mathrm{~min} \\
\text { 3. } 1 \times ; 5 \mathrm{~min} \\
\text { 4. } 5 \mathrm{~min}\end{array}$ & $\begin{array}{l}\text { 2. Not reported } \\
\text { 3. Averaging } \\
\text { 4. OxyHb; CBV; COV } \\
\text { 5. Entire task phase }\end{array}$ \\
\hline Suzuki et al. ${ }^{62}$ & $\begin{array}{l}\text { - Healthy, young adults } \\
(n=9 ; 28.1 \pm 7.4,22 \text { to } 46) \\
\text { - Walking at different speeds }\end{array}$ & $\begin{array}{l}\text { 2. First } 13 \mathrm{~s} \\
\text { 3. } 3 \times ; 90 \mathrm{~s} \\
\text { 4. } 30 \mathrm{~s} \text { between trials }\end{array}$ & $\begin{array}{l}\text { 1. N/A (arbitrary unit) } \\
\text { 2. HPF at } 0.03 \mathrm{~Hz} \\
\text { 3. Linear interpolation; baseline } \\
\text { correction; averaging } \\
\text { 4. Oxy- and deoxyHb; regional cortical } \\
\text { activation ratio (oxy } \mathrm{Hb} \text { of the specific } \\
\text { channel divided by oxyHb of all } 42 \\
\text { channels multiplied by } 100 \text { ) } \\
\text { 5. } 13.5 \mathrm{~s} \text { in task phase }\end{array}$ \\
\hline Suzuki et al. ${ }^{67}$ & $\begin{array}{l}\text { - Healthy, young adults } \\
(n=7 ; 31.3 \pm 4.8,24 \text { to } 45) \\
\text { - Walking with vs. without verbal } \\
\text { preadvice }\end{array}$ & $\begin{array}{l}\text { 1. Quiet standing } \\
\text { 2. } 10 \mathrm{~s} \text { before walking } \\
\text { 3. } 4 \times ; 40 \mathrm{~s} \\
\text { 4. } 10 \text { to } 25 \mathrm{~s} \text { between trials } \\
\text { (randomized order) }\end{array}$ & $\begin{array}{l}\text { 1. Not relevant (arbitrary unit) } \\
\text { 2. HPF at } 0.03 \mathrm{~Hz} \\
\text { 3. Baseline normalization; averaging } \\
\text { 4. Oxy- and deoxyHb } \\
\text { 5. First } 10 \mathrm{~s} \text { of task phase }\end{array}$ \\
\hline Takeuchi et al. ${ }^{89}$ & $\begin{array}{l}\text { - Young healthy adults } \\
\quad(n=16 ; 25.9 \pm 4.4,20 \text { to } 33) \\
\text { - Healthy older adults } \\
\quad(n=15 ; 71.7 \pm 3.3,65 \text { to } 78) \\
\text { - Walking vs. walking with smartphone }\end{array}$ & $\begin{array}{l}\text { 1. Walking } \\
\text { 2. } 30 \mathrm{~s} \\
\text { 3. } 15 \times ; 10 \mathrm{~s} \\
\text { 4. No rest }\end{array}$ & $\begin{array}{l}\text { 1. Constant value (no details reported) } \\
\text { 2. Spike rejection (artifact with more than } \\
3 \mathrm{SD} \text { ); } 5 \mathrm{~s} \text { moving average; bandpass } \\
\text { filter (LPF at } 0.5 \mathrm{~Hz} \text {; HPF at } 0.01 \mathrm{~Hz} \text { ) } \\
\text { 3. Baseline normalization; averaging } \\
\text { 4. OxyHb } \\
\text { 5. Entire task phase }\end{array}$ \\
\hline Takakura et al. ${ }^{96}$ & $\begin{array}{l}\text { - Healthy young adults } \\
(n=11 ; 33.4 \pm 7.4) \\
\text { - SOT conditions }\end{array}$ & $\begin{array}{l}\text { 1. Quiet standing } \\
\text { 2. } 20 \mathrm{~s} \text { before task } \\
\text { 3. } 3 \times ; 20 \mathrm{~s} \\
\text { 4. Few minutes after } 3 \text { trials }\end{array}$ & $\begin{array}{l}\text { 1. Constant value (1.0) } \\
\text { 2. Bandpass Fourier filter ( } 0.01 \text { to } 0.1 \mathrm{~Hz}) \\
\text { 3. Averaging } \\
\text { 4. OxyHb } \\
\text { 5. Entire task phase }\end{array}$ \\
\hline
\end{tabular}


Table 1 (Continued).

\begin{tabular}{|c|c|c|c|}
\hline \multirow{5}{*}{ Verghese et al. ${ }^{83}$} & - Older adults $(n=166 ; 75 \pm 6.1)$ & 1. Quiet standing & 1. Constant value (6.0) \\
\hline & \multirow[t]{4}{*}{ - NW vs. DTW vs. standing } & $\begin{array}{l}\text { 2. } 10 \mathrm{~s} \text { (counting silently } \\
\text { forward in steps of } 1 \text { ) }\end{array}$ & $\begin{array}{l}\text { 2. LPF at } 0.14 \mathrm{~Hz} \text {; noisy channels } \\
\text { excluded (dark current condition or } \\
\text { saturation); visual inspected }\end{array}$ \\
\hline & & $\begin{array}{l}\text { 3. } 3 \times \text { walking loops }(=6 \times \\
\text { straight walks a } 14 \mathrm{ft} .) / \\
\text { standing for } 30 \mathrm{~s} \text { ) }\end{array}$ & 3. Baseline normalization; averaging \\
\hline & & 4. "Short break" reported & 4. OxyHb \\
\hline & & & 5. Entire task phase \\
\hline \multirow[t]{5}{*}{ Wang et al. ${ }^{104}$} & $\begin{array}{l}\text { - Healthy young adults } \\
\quad(n=22 ; 24.4 \pm 1.6)\end{array}$ & 1. Sitting (eyes closed) & $\begin{array}{l}\text { 1. Age-dependent constant value } \\
(\mathrm{WL}: 760 \mathrm{~nm}=5.91 ; \mathrm{WL}: 850=5.40)\end{array}$ \\
\hline & $\begin{array}{l}\text { - Healthy older adults } \\
\quad(n=39 ; 70.5 \pm 7.7)\end{array}$ & 2. $20 \mathrm{~min}$ & 2. Bandpass filter (0.005 to $2 \mathrm{HZ}$ ) \\
\hline & \multirow{3}{*}{$\begin{array}{l}\text { - Standing connectivity differences } \\
\text { healthy young and healthy old adults }\end{array}$} & 3. $1 \times ; 10 \mathrm{~min}$ & 3. Wavelet phase coherence analysis \\
\hline & & 4. No rest & 4. OxyHb \\
\hline & & & 5. Entire task time \\
\hline
\end{tabular}

Abbreviations: deoxy Hb, deoxygenated hemoglobin; DTS, dual-task standing; DTW, dual-task walking; ft., feet; HC, healthy controls; HPF, highpass filter; HRF, hemodynamic response function; LPF, low-pass filter; MDL, minimum description length; MRI, magnetic resonance imaging; N/A, not applicable; NW, normal walking; NGA, neurological gait abnormalities; oxyHb, oxygenated hemoglobin; PCA, principal component analysis; RAW, robot assisted walking; SOT, sensory organization test; ST, stepping; TOI, tissue oxygenation index; and vs., versus.

\subsection{Differential Path Length Factor}

The differential path length factor (DPF) is a scaling factor that specifies how many times the detected light has traveled farther than the source-detector separation through the brain. ${ }^{109,110}$ In 21 studies, constant DPF values were used $^{63,69-71,73-76,78,81-85,87-89,93,96,105,108}$ whereas nine studies used age-dependent DPF values. ${ }^{57,65,77,79,80,90,98,99,104}$

For constant DPF, values of $1.0,{ }^{96} 5.9,{ }^{78} 5.93,{ }^{70,71} 5.94,{ }^{88}$ and $6.0^{73-76,81-85,87,93,105,108}$ were used, while age-dependent DPF values were calculated according to the formula ( $\mathrm{DPF}=4.99+$ $0.067 \times$ age $\left.^{0.814}\right) .{ }^{57,65,77,79,80,90,98,99}$ An overview on used DPF values is provided in Fig. 2(b). In other studies, arbitrary units, ${ }^{53-56,58-62,64,67,72,94,95,100-102,107}$ tissue oxygenation index [TOI; the ratio of oxyHb to total hemoglobin (sum of oxy- and deoxyHb)], ${ }^{66,86}$ image reconstruction,,${ }^{91,92,97,103}$ or absolute values ${ }^{68}$ were used, which are not dependent on specific DPF values.

\subsection{Data Processing: Signal Filtering and Movement Artifact Removal}

Twenty-one studies applied a low-pass filter (LPF) to their data, ${ }^{54,57,65,71-75,77,79,81-83,85,88,90,93,98-101} 14$ studies used a highpass filter (HPF) $)^{53,55,56,59-62,67,94,95,100-102,107}$ and 5 studies used a bandpass filter. ${ }^{76,80,89,96,104}$ Most studies applied an LPF with a cut-off frequency around $0.1 \mathrm{~Hz}^{71,73-75,77,80-85,88,90,93,98,99,108}$ whereas some studies used an LPF with a cut-off frequency at $0.05,{ }^{72} 0.67,{ }^{57,65} 0.5,{ }^{100,101}$ and $1 \mathrm{~Hz}^{54}$ (for an overview see Table 1). Eight studies applied an HPF with a cut-off frequency at $0.03 \mathrm{~Hz},{ }^{53,59-62,67,95,107}$ six studies at $0.01 \mathrm{~Hz},{ }^{54-56,80,100,101}$ and one study at $0.05^{94}$ or $0.001 \mathrm{~Hz} .{ }^{108}$ Furthermore, eight studies used the moving average method ${ }^{55-57,64,68,89,100,101}$ to smooth their data. Filter methods based on principal component analysis (PCA) were conducted in six studies ${ }^{55,56,73,76,100,102}$ and a spike artifact correction was used in three studies. ${ }^{68,76,89}$ Few studies
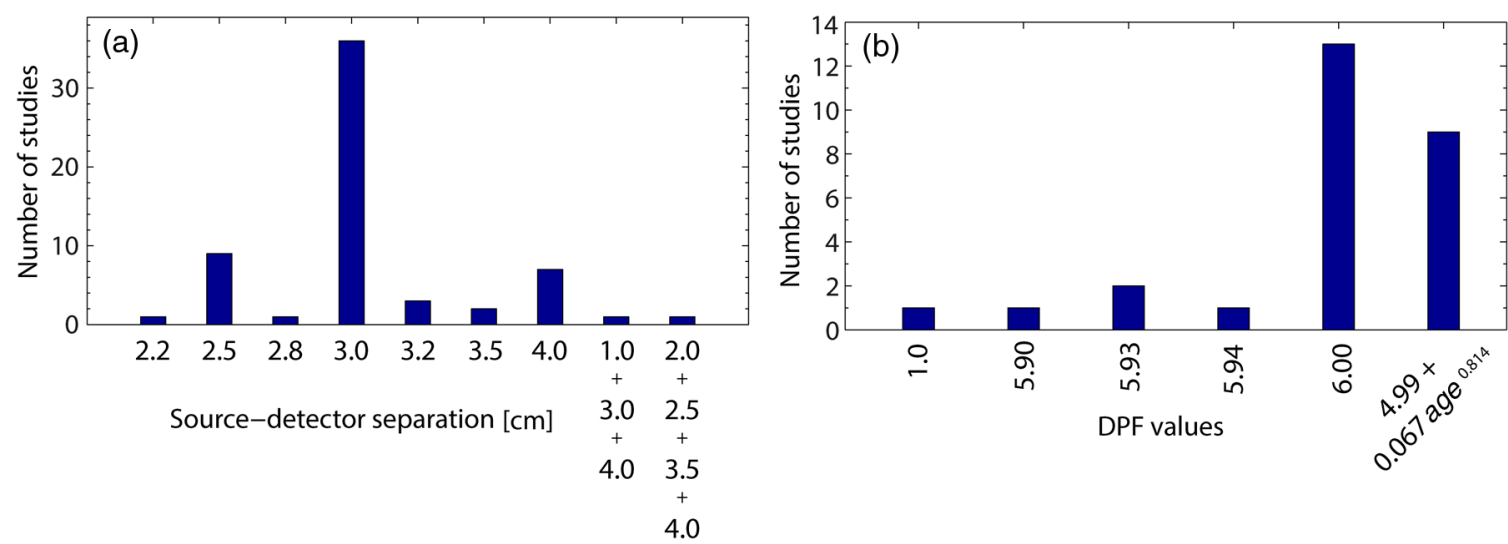

Fig. 2 Overview on (a) used source-detector separation and (b) DPF values in the reviewed studies. 
applied HRF filter, ${ }^{105}$ an autoregressive model with prewhitened iterative reweighted least square algorithms, ${ }^{103}$ wavelet filter, ${ }^{80,105,106}$ Gaussian smoothing, ${ }^{106}$ and correlation-based signal improvement (CBSI) ${ }^{64,80} \mathrm{~A}$ visual inspection of data was reported in 13 studies. ${ }^{68,74,75,81-85,88,93,97,108,111}$

\subsection{Data Processing: Correction for Physiological Artifacts}

One study applied short separation channels ${ }^{54}$ and one study used multidistance measurements ${ }^{68}$ to correct for superficial blood flow. For multidistance measurements or short separation channels, normally lower source-detector separation $(<1.5 \mathrm{~cm})$ is chosen, which is used to probe extracerebral noise. Furthermore, the following additional physiological parameters were measured to take into account systemic physiological artifacts: (1) heart rate, ${ }^{53,57,59-62,71,87,98,99,107}$ (2) blood pressure, ${ }^{53,54,57,59-62,71,107}$ and (3) arterial oxygen saturation. ${ }^{59-62,107}$ The usage of filter methods based on PCA, which can be useful for the correction of motion and physiological noise, was used in six studies. $55,56,73,76,100,102$

\subsection{Data Processing: Final Data Processing and Statistical Analysis}

Twenty-three of the reviewed studies used a baseline correction $^{53,55,56,58-62,64,65,69-71,77,79-81,87,90,96,100,101,105}$ and 14 studies conducted a baseline normalization. ${ }^{53,54,67,73-75,82-}$ $85,89,93,101,108$ Furthermore, almost all studies computed an average of (1) all trials and (2) across the channels of a specific ROI. ${ }^{53-65,68-77,80-85,87,89-91,93-96,98-101,103,107}$

In addition, linear interpolations were used in the studies of Miyai et al. and Suzuki et al. ${ }^{59-62,107}$ A method based on moving standard deviation and spline interpolation was applied by Beurskens et al. ${ }^{105}$ Three studies applied discrete cosine transform terms. ${ }^{91,92,97}$

Canonical hemodynamic response function was conducted in two studies ${ }^{105,106}$ that examined cortical activation during walking. Studies researching postural tasks used either a gamma hemodynamic response function ${ }^{91,92,95,102,103}$ or a Gaussian hemodynamic response function. ${ }^{94} \mathrm{~A}$ wavelet coherence analysis was used in one study. ${ }^{104}$

Five studies divided their task phase in different time periods, ${ }^{58,68,76,86,90} 18$ studies used predetermined time intervals inside the task phase, ${ }^{53,54,57,59-62,65,67,69-71,73,93,94,98-100}$ and 24 studies used the entire task phase for analysis. ${ }^{55,56,63,64,72,74,75,77,80,82,83,85,87,89,91,92,96,97,101-103,106-108}$

The statistical analysis was performed in 47 studies with parametric $^{53,54,56-70,73-77,79,80,82-89,91-99,103-108}$ and in one study with nonparametric methods. ${ }^{81}$ Eight studies used parametric and nonparametric methods ${ }^{55,71,72,78,90,100-102}$ (see Table 1).

\subsection{Markers for the Assessment of Cortical Activation}

The majority of reviewed studies used changes of oxyHb to assess brain activation. ${ }^{53,57-62,68,70-75,77-80,82-85,89,90,93-96,101,104,106}$ Furthermore, 21 studies used both oxyHb and deoxyHb to quantify the activation of the region of interest. ${ }^{54-56,63-}$ $65,67,69,81,88,91,92,97-100,102,103,105,107,108$ Only Clark et al. ${ }^{66,86}$ used the TOI, which is the ratio of oxygenated to total tissue hemoglobin, to evaluate cortical activation. In addition, $\mathrm{Lu}$ et al. ${ }^{76}$ used $\mathrm{Hb}$ diff (oxyHb - deoxyHb) for the quantification of cortical activation. Furthermore, one study used a cortical activation ratio ${ }^{62}$ to measure brain activation (for an overview see Table 1).

\section{Results: Main Findings of the Studies}

In the following sections, we will provide an overview about the main findings of the reviewed studies. The results section is divided into outcomes of walking and postural tasks.

\subsection{Walking}

Walking was associated with a higher activation of prefrontal cortex (PFC), ${ }^{53,54,57,58,67}$ presupplementary motor area, ${ }^{53,67}$ premotor cortex (PMC), ${ }^{53,106}$ supplementary motor area (SMA), ${ }^{53-}$ $55,58,67,106,107$ and sensorimotor cortex (SMC). ${ }^{53,58,67,106}$ A higher PFC activation was observed in persons with low gait capacity, ${ }^{53}$ high perceived stress ${ }^{84}$ high perceived fatigue ${ }^{85}$ high risk of falling, ${ }^{83}$ ataxic gait, ${ }^{70,71}$ and patients with Parkinson's disease $^{80,90}$ during walking. Moreover, higher activation of precentral gyrus (PrG), postcentral gyrus (PoG), and superior parietal lobule (SPL) was observed in children with cerebral palsy ${ }^{56}$ and in stroke patients in the nonaffected hemisphere in the PFC, ${ }^{58}$ SMA, ${ }^{58,61}$ and SMC. ${ }^{61}$ During dual-task walking (e.g., walking and solving an additional cognitive or motor task), the PFC exhibited an enhanced activation in stroke patients, ${ }^{65}$ patients with multiple sclerosis, ${ }^{82}$ patients with Parkinson's disease, ${ }^{81}$ obese adults, ${ }^{87}$ older adults with mild cognitive impairments, ${ }^{72}$ old adults with mobility deficits, ${ }^{66,86}$ and healthy older $^{63,65,73-75,82}$ and young adults. ${ }^{57,63,64,73,76,77}$ In comparison to young adults, older adults exhibited a higher ${ }^{73}$ or similar ${ }^{63}$ PFC activation during dual-task walking. The activation of PFC during dual-task walking was associated with the performance in motor tasks, ${ }^{75,77,89,105}$ cognitive tasks, ${ }^{75,77,89}$ and neuropsychological tests. ${ }^{72}$ In single task walking, PFC activation positively correlated with the neuropsychological performance in healthy older persons ${ }^{68}$ and with motor performance in neurologically diseased persons. ${ }^{70,71}$ A decrease in PFC activation was observed in younger adults while walking and solving a working memory task ${ }^{79,88}$ and in healthy seniors while solving a complex visual task. ${ }^{105}$ Interestingly, the activation of PFC in older adults is decreased after a motor intervention ${ }^{68}$ and when textured insoles were used or barefoot walking was conducted. ${ }^{66}$ In contrast, the inpatient intervention in stroke patients enhanced PMC activation during walking. ${ }^{60}$ Additionally, an increase of motor complexity due to the increase in walking speed led to a pronounced activation of PFC, ${ }^{62} \mathrm{SMA}^{53}$ and Broca area, ${ }^{64}$ whereas a decrease of motor complexity due to body weight support induced a decrease in SMC activation. ${ }^{59}$

\subsection{Postural Tasks}

In balance tasks, the activation of PFC, ${ }^{91,98,99}$ SMA, ${ }^{101,102}$ and superior temporal gyrus ${ }^{97}$ was modulated by task difficulty and by age-related processes. ${ }^{104}$ Furthermore, an increased PFC activation was observed during standing in young adults with postconcussion symptoms, ${ }^{108}$ in patients with Parkinson's disease ${ }^{93}$ or in stroke patients' in the affected ${ }^{95,102}$ and unaffected hemisphere. ${ }^{95}$ Furthermore, stroke patients showed a stronger activation in PMC and parietal areas concerning the unaffected hemisphere. ${ }^{95}$ After the rehabilitation program, the same patients showed a decreased activation of PMC and parietal areas but a bilateral increase in PFC and SMA activations. ${ }^{102}$ 
During the SOT, different sensory information changes the functional connectivity of brain areas ${ }^{96,103}$ and induced activation changes especially in superior marginal gyrus, ${ }^{92,96}$ operculum, ${ }^{96}$ temporal-parietal areas, ${ }^{103}$ and occipital regions. ${ }^{103}$ Additionally, correlation between balance performance and the activation of PFC $^{95,102}$ and SMA was observed. ${ }^{95,100,102}$

\section{Discussion}

fNIRS is a relatively new neuroimaging technique that has attracted attention in scientists who examine neuromotor control. This resulted in a considerable magnitude of published studies. However, a summarization and evaluation that can help to improve future experimental protocols was still required. In the first part of the discussion section, we will discuss the findings about study designs, fNIRS configurations and data processing steps to come closer to more standardized protocols that are not available at this moment. ${ }^{27,112}$ In the second part, the main findings of the reviewed studies are discussed.

\subsection{Discussion: Methodology}

\subsubsection{Baseline condition and duration}

The majority of studies with walking or postural tasks assessed baseline brain activation in quiet standing. Interestingly, Holtzer et al. ${ }^{74,75}$ used a silent counting task to avoid mind wandering. Mind wandering occurs up to $50 \%$ of the waking hours ${ }^{113}$ for instance during driving ${ }^{114,115}$ especially when perceptual requirements are low. ${ }^{116}$ Moreover, the wandering of the mind is characterized by the processing of task unrelated thoughts such as worrying about the past or future, ${ }^{117}$ which evokes a stronger activation of default networks ${ }^{118}$ and hence changes the activation in PFC areas. ${ }^{119,120}$ In addition, it was shown by Durantin et al. ${ }^{120}$ that fNIRS is sensitive to detect mind wandering. Based on these assumptions, it is possible that mind wandering influences the cortical activation during baseline (and maybe motor control) affecting further analyzation processes. Hence, it might be advantageous to use the approach of Holtzer et al. ${ }^{74,75}$ which eventually minimizes the detrimental effect of mind wandering on cortical activation and leads to a more standardized baseline assessment. However, before the usage of this simple counting task can be recommended, further research should investigate its influence on cortical activation patterns including examination of enhanced reproducibility.

\subsubsection{Number and duration of trials and rest phases}

Our results revealed that the number of trials and their durations varied across the studies evaluating walking or postural tasks. The most common time interval was set to $30 \mathrm{~s}$. However, we are unaware of a study investigating the influence of measurement strategy (e.g., required number of trials to achieve a sufficient reproducibility). Hence, further methodological investigations to optimize fNIRS measurement protocols are needed. Moreover, the duration and number of the trials depend on the aim of the study. Longer measurement durations may be useful to study the contribution of different areas in the temporal course of movement execution. In contrast, longer measurement durations could result in motor fatigue. Motor fatigue does diminish performance for example in postural tasks ${ }^{121-126}$ and would hence change underlying motor control processes. This again could potentially evoke altered hemodynamic responses, which were observed after cognitive fatigue. ${ }^{127}$ However, research examining the interplay between a specific gross motor task and hemodynamic responses as a function of physical fatigue level has not been conducted yet.

Another interesting point influencing the trial duration is the combination of analysis methods. From a movement scientific view, the analysis of gait features (especially gait variability and stability) gives an insight in the central organization of motor control processes ${ }^{128-131}$ and those are useful to detect risk groups such as fallers. ${ }^{132,133}$ To reliably assess gait variability or stability, a larger number of strides is required ${ }^{134,135}$ and as a consequence, a sufficiently long time period (in which an adequate number of strides can be undertaken) of the trial duration has to be recorded. The rest phase durations in included studies have varying temporal ranges. In general, empirical evidence suggests that refraction time or time with reduced responsiveness lasted for almost the same duration as stimulus time. ${ }^{136}$ Hence, we recommend to include intertrial rest intervals with at least the same duration as the task period, especially in block design studies.

\subsubsection{Source-detector separation}

The separation of source to detector is one important aspect for penetration depth ${ }^{27,34}$ and the influence of extracerebral signals. ${ }^{34,137}$ Our results indicated that $3 \mathrm{~cm}$ was the most commonly used distance in the reviewed studies. In the literature, different recommendations about optimal source-detector separation exist. While some authors recommend $4 \mathrm{~cm},{ }^{34}$ other collectives recommend $3 \mathrm{~cm} .{ }^{138,139}$ In addition, especially in children or infants shorter interoptode distance $(>2.0 \mathrm{~cm})$ is recommended for usage. ${ }^{22,139}$ The issue of the optimal separation between source and detector is a controversial debate because different third variables such as different colors of the participant's skin and/or hair used wavelengths and head size could influence penetration depth. ${ }^{34,140}$ Furthermore, the varying thickness of scalps, skulls, and cerebrospinal fluids in individuals and cortical regions ${ }^{141-143}$ could influence the penetration depth and the sensitivity to hemodynamic changes in cortical layer. $^{142-144}$

Remarkably, a longer source-detector separation leads to a greater contribution of cerebral than extracerebral layer to obtain hemodynamics signals. ${ }^{145-148}$ The penetration depth of light is less than half of the interoptode distance ${ }^{147}$ causing short channel distances to cover only signals from noncerebral compartments. ${ }^{137,141}$ For instance, at the source-detector separation of $3 \mathrm{~cm}$, the contribution of the gray matter to the light absorption is estimated to range from about $20 \%$ to $30 \%$. ${ }^{149}$ Moreover, Kohri et al. ${ }^{150}$ observed that at source-detector separation of 2,3 , and $4 \mathrm{~cm}$, the cerebral tissue contributes to $33 \%, 55 \%$, and $69 \%$ to the optical signal. Hence, we recommend that the source-detector separation should be greater than $3 \mathrm{~cm}$ to enhance the contribution of cerebral cortical layer to the optical signal.

\subsubsection{Placement of optodes}

The majority of the studies used the 10 to 20 EEG systems to place the optodes. This standardized location system ensures the comparability among the different studies. The additionally used 3-D digitizer or individual MRI scan improves the registration of channels to specific brain areas. Based on the data we recommend for optode placement the usage of the 10 to $20 \mathrm{EEG}$ systems to ensure the comparability among studies. 


\subsubsection{Differential path length factor}

Our results show that most studies used constant DPF with a value of 6 . The usage of a constant DPF value seems not always appropriate because the brain undergoes age-related changes of gray and white matter, ${ }^{151,152}$ intracranial volume, ${ }^{153}$ and cerebral volume as well as blood flow ${ }^{154}$, which may affect DPF. ${ }^{155}$ Furthermore, methodological studies show that DPF values are (1) age-dependent and subject-specific, ${ }^{110,155,156}$ (2) wavelength-dependent, ${ }^{110,155,157}$ and (3) cortex region-dependent. ${ }^{110,155,158-160}$ Hence, it seems favorable to calculate specific DPF values to enhance the measurement accuracy in age-groups in which formulas to calculate age-specific DPF values are available (adults under 50 years). ${ }^{110,155}$ Otherwise, "arbitrary units,"161 TOI, ${ }^{162-164}$ or absolute values ${ }^{137,163,165}$ could be used since those do not depend on a specific DPF value. In addition, it is suggested that the calculation of effect sizes is useful to deal with the DPF issue. ${ }^{166}$ However, additional research is strongly needed that provides a formula to calculate DPF values for specific age-groups (adults older than 50 years) dependent on wavelength and cortex region. In our opinion, the optimal approach to quantify DPF, taking the dependency of DPF regarding subject, age, wavelength, and cortex region into account, is the direct quantification of DPF using frequency or time-domain NIRS.

\subsubsection{Data processing: signal filtering and movement artifact removal}

In sum, either LPFs or HPFs were commonly applied in the reviewed studies to remove noise and drifts. Most of the studies used a cut-off frequency for LPF around $0.1 \mathrm{~Hz}$ and HPF around $0.01 \mathrm{~Hz}$. The reviews of Brigadoi et al., ${ }^{167}$ Cooper et al., ${ }^{168}$ and Gervain et al. ${ }^{40}$ recommended to use a bandpass filter (consisting of both LPFs and HPFs) with cut-off frequencies at 0.5 (LPF) and $0.01 \mathrm{~Hz}$ (HPF). The bandpass filtering should be used carefully to avoid accidental removal of stimulusdependent hemodynamic response signals. ${ }^{111}$ Hence, a higher cut-off frequency at $0.5 \mathrm{~Hz}$ (LPF) in conjunction with other more sophisticated filter methods is recommended to be used for the removal of movement and physiological noise. ${ }^{111,167,168}$ Different methods such as PCA, ${ }^{169-171}$ task-related component analysis, ${ }^{172-174} \mathrm{CBSI},{ }^{175}$ wavelet-based filters, ${ }^{171,176-179}$ autoregressive algorithm-based filters, ${ }^{180}$ Kalman filter, ${ }^{181}$ and Wiener filter ${ }^{182}$ are proposed for the filtering of fNIRS data. Interestingly, Nozawa et al. ${ }^{183}$ suggested that effectiveness of motion correction filter methods depends on subject and task. However, reviews comparing a variety of filter methods recommend the additional application of wavelet filter ${ }^{167,168}$ or spline technique. ${ }^{168}$ These filter methods were occasionally applied in reviewed studies ${ }^{80,105,106}$ leaving potential to optimize the filtering processes in further studies. Based on these assumptions, we recommend the usage of a bandpass filter and wavelet filter to reduce motion artifacts. If there are sudden shifts in the data (baseline shift), the approach developed by Scholkmann et al. ${ }^{184}$ can be useful to remove them.

\subsubsection{Data processing: correction for physiological artifacts}

Twelve studies recorded physiological signals such as heart rate, blood pressure, or arterial oxygenation saturation parallel to the fNIRS signals. Task-related systematic changes in heart rate, respiration rate, or blood pressure are known to influence the fNIRS signal and may cause false-positive results. ${ }^{45}$ For instance, often unconsidered factors such as adding of speech as a task (e.g., in dual-task paradigms) lead to changes in partial pressure of end-tidal carbon dioxide, which influences cerebral hemodynamics and masked neuronal-induced activity changes. ${ }^{185,186}$ Hence, to improve the accuracy of fNIRS, the recording and elimination of systemic physiological changes seems necessary. ${ }^{45,187,188}$ The signals of additional physiological measures could be useful for filtering of fNIRS signal ${ }^{189,190}$ or to ensure the absence of systematic physiological differences among the experimental conditions. ${ }^{87}$ In addition, some measures such as heart rate variability could be used to study the interplay between the central (fNIRS) and the autonomic (e.g., heart rate variability) nervous system. ${ }^{120,191}$ Furthermore, filter methods based on PCA and independent component analysis, which were applied in six studies,${ }^{55,56,73,76,100,102}$ could be used to remove movement-related ${ }^{167}$ or physiological artifacts. ${ }^{169,170,192-195}$ In addition to the other filter methods, ${ }^{196,197}$ a more "direct" approach to reduce extracerebral noise is the use of short separation channels or multidistance technique ${ }^{198-200}$, which were applied in only two of the reviewed studies. ${ }^{54,68}$ Short separation channels have a small distance between source and detector to record extra cerebral signals, such as superficial blood flow. ${ }^{141,198,201}$ These extracerebral signals are used to filter the remaining fNIRS data. Previous studies revealed that the application of short separation channels is powerful in reducing extracerebral noise ${ }^{141,145,200,202-208}$, which contaminates fNIRS signals. ${ }^{45,199,201,209-214}$ The optimal distance between short separation channels varied across different cortex regions ${ }^{141,202}$ but should be generally $<1 \mathrm{~cm}$ for measurement on the head of adult humans. Hence, further development and implementation of short separation channels (multidistance technique) could enhance the accuracy of fNIRS measurements and have to be considered whenever technically possible.

\subsubsection{Data processing: final data processing and statistical analysis}

Most studies used baseline normalization and baseline correction to circumvent the influence of different path lengths factors. ${ }^{166}$ Furthermore, averaging of channels across trials and in specified ROIs was common practice in the reviewed studies.

Some studies divided their task phase in different time periods, which seems useful for studying the contribution of cortical areas in different temporal periods during task execution. Therefore, attention should be paid to the temporal delay of $\sim 2$ to $5 \mathrm{~s}$ in hemodynamic response. ${ }^{69,107,139}$

The majority of the reviewed studies used simple statistics based on processing mean values over the task period. This approach, however, tends to result in a loss of acquisition of information because it does not consider the temporal shape of the fNIRS signal. ${ }^{192}$ Hence, some authors suggest that the analysis of fNIRS data with general linear models is more favorable. ${ }^{192,215}$ However, the choice of the statistical analysis methods should depend on the research question and the experimental design. ${ }^{216}$ For instance, in an event-related design, the application of a general linear model is a valid technique ${ }^{216}$ whereas simple statistics might also be appropriate (and commonly used ${ }^{192}$ ) especially in studies utilizing block designs. ${ }^{55,56,59-62,107,217}$ The majority of reviewed studies used 
parametric methods for statistical data analysis. In fNIRS studies, the assumptions for parametric tests are sometimes violated (e.g., normal distribution due to small sample size); therefore, nonparametric tests are a considerable option. ${ }^{218,219}$ Moreover, nonparametric tests are more robust and less influenced by outliers or nonnormal distributed data ${ }^{220-222}$ and are recommended to use in fNIRS studies. From another point of view, in neuroscience, multiple experimental conditions (crossed) or multiple observations per condition (nested) were used. ${ }^{223,224}$ Furthermore, different categorical or continuous confounding variables have to be considered (e.g., gait speed, education, and gender) and/or data were unbalanced or incomplete, which makes it necessary to use advanced statistical methods. ${ }^{223,225}$ To account for those problems, linear mixed-effect models can be used. ${ }^{10,224-226}$ However, statistical methods should be chosen carefully considering the experimental design and distribution of recorded data. A further description of statistical methods for fNIRS data is given in the reviews of Tak and $\mathrm{Ye}^{192}$ and Kamran et al. ${ }^{227}$

\subsubsection{Markers for the assessment of cortical activation}

The majority of reviewed studies used only oxyHb for the quantification of cortical activation since a change in oxyHb is assumed to be a more robust marker of changes in regional cerebral blood flow than changes in deoxyHb. ${ }^{160,228,229}$ However, this procedure seems questionable because neuronal activity is not just mirrored in an increase of oxyHb but also in a decrease in deoxyHb in healthy adults. ${ }^{30,230}$ Furthermore, an enhanced level of physiological noise is more prominent in oxyHb signals ${ }^{30}$ and the decrease in deoxyHb is related to an increase in BOLD contrast obtained in $\mathrm{fMRI}^{231,232}$, which supports the validity of the evaluation of deoxyHb changes. In pathological states, neurovascular coupling might perhaps be impaired, which results in altered concentration changes in deoxyHb during neural activity. ${ }^{230}$ Lindauer et al. ${ }^{230}$ assumed that in some pathological states, an increase in deoxyHb may reflect neural activity. Based on the mentioned assumptions, it seems favorable to report at least oxyHb as well as deoxyHb to assess task-dependent activity. ${ }^{30,45,165}$

\subsection{Discussion: Main Findings}

\subsubsection{Walking}

Evidence from neuroimaging studies point out that two distinct supraspinal locomotor networks are responsible for the control of walking and standing ${ }^{1,233-237}$ (see Fig. 3). The direct locomotor network consists of the primary motor cortex (M 1) and the cerebellar locomotor region and is potentially activated in the absence of pathologies or challenging situations. ${ }^{235}$ In the indirect locomotor pathway, the neuronal commands are transmitted via PFC and SMA to the basal ganglia and subthalamic as well as mesencephalic locomotor regions. ${ }^{233-237}$ The indirect locomotor pathway becomes activated when the automatic execution of walking is impaired (e.g., in challenging situations) and compensatory mechanisms are necessary. ${ }^{44,238}$ This assumption is supported by findings of our reviewed fNIRS studies, which reported more pronounced activation in prefrontal structures in (1) in adults during dual-task walking, $57,63,63,66,69,72-74,77,86,89$ (2) in adults during fast walking, ${ }^{53,64}$ (3) in obese persons, ${ }^{87}$ (4) in individuals with low gait capacity during fast walking, ${ }^{53}$ (5) in older adults with high level of perceived fatigue ${ }^{85}$ or stress, ${ }^{84}(6)$ in old adults with increased fall risk, ${ }^{83}$ and (7) in neurological patients. ${ }^{58,70,71,75,80,82,90}$ Remarkably, the PFC activation in neurological patients correlates with their step widths, ${ }^{71}$ which again (1) is associated with balance control $^{239}$ and (2) serves as a predictor of falls. ${ }^{240}$ Furthermore, correlations between cortical activation and motor performance, ${ }^{55,56}$ especially obvious in dual-task walking conditions, ${ }^{76,77,89,105}$ was observed. This reinforces the important role of cortical areas in motor control. Moreover, the reduction of PFC activity after a motor-cognitive intervention program

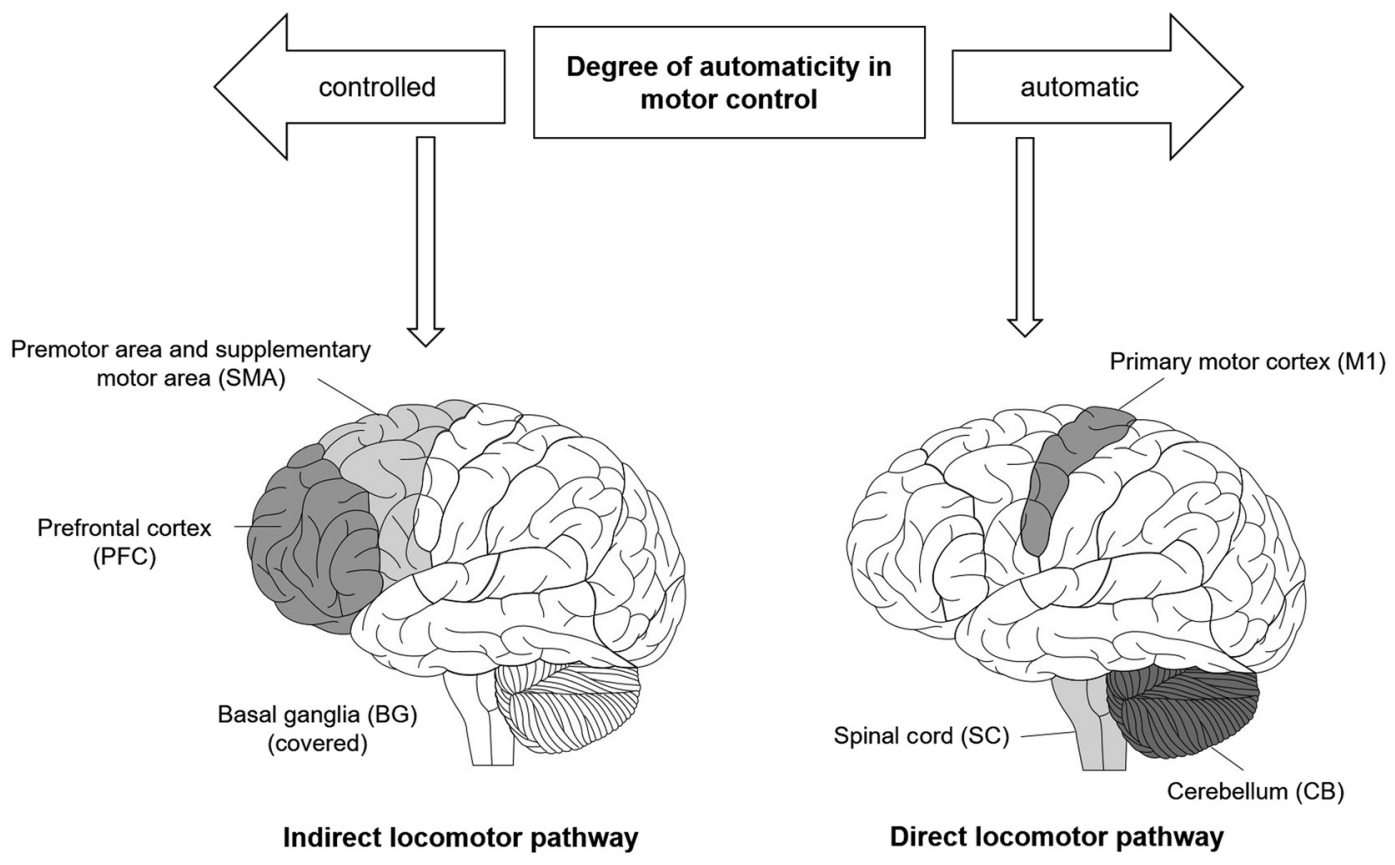

Fig. 3 Schematic illustration of the indirect and direct locomotor pathways as a function of the degree of automaticity in motor control. 
(lasting 8 weeks) ${ }^{68}$ perhaps originated from the shift toward a more automatic control of locomotion relying on the enhanced usage of direct locomotor pathway via M1, cerebellum, and spinal cord. . $233,234,238$

However, premotor areas and the SMA play a role in different cognitive processes ${ }^{241-243}$ and were activated as a function of task difficulty in a variety of cognitive domains. ${ }^{244-246}$ Hence, the phenomenon of a more pronounced activation of premotor areas (as part of indirect locomotor pathway) in diseased cohorts (or during challenging motor tasks) is perhaps not fully attributable to motor task complexity but partly also to general task complexity.

However, the decrease in PFC activity in a complex visual $\operatorname{task}^{105}$ or difficult working memory tasks during walking ${ }^{79,88}$ may not be induced by the shifts in locomotor pathways but rather originate from the prioritization of task-relevant areas as consequence of the limited resources of the brain. ${ }^{247}$ While those three studies focused only on PFC activity, it is difficult to draw a final conclusion about potentially underlying cortical processes in other areas. Hence, to elucidate the mechanisms with respect to task prioritizations, we require further research $^{248,249}$ including the simultaneous assessment of more cortical structures (e.g., motor areas).

For the design and monitoring of rehabilitative interventions, fNIRS could be a promising tool. ${ }^{42}$ For instance, the SMC activity decreases during weight-supported walking in stroke patients $^{59}$ and could be a hint that weight supports lower task complexity. ${ }^{250}$ Interestingly, a verbal preadvice ${ }^{67,94}$ or the usage of mechanical assistance during walking ${ }^{61,106}$ increases central nervous load. These findings could be useful to create tailored rehabilitation programs that consider mental load as variable for workload assessment.

\subsubsection{Postural tasks}

As pointed out for walking, neural control of posture is realized via direct or indirect pathway ${ }^{251}$ which are shown in Fig. 3. Our results reveal that the PFC activation is enhanced in (1) neurological patients during standing ${ }^{93}$ or during postural perturbations $\mathrm{s}^{95,102}$ and (2) healthy adults during challenging balance tasks. ${ }^{91,98,99}$ These findings and the observations that PFC activity and SMA are associated with balance measures ${ }^{95,100,102}$ support the notion that indirect locomotor pathway is crucial for neuromotor processes in nonautomatized challenging situations.

Additionally, altered sensory information evoked by the execution of SOT induces a higher activation especially in STG. ${ }^{92,96}$ The STG is associated with (1) the control of more difficult balance tasks, ${ }^{97}(2)$ the integration of vestibular information, ${ }^{252-254}$ and (3) the spatial orientation. ${ }^{255}$ So far, the mentioned studies did include only young participants. ${ }^{92,96}$ While aging changes the contribution of somatosensory, vestibular, and visual system in balance tasks, ${ }^{256}$ it seems necessary to enlarge existing knowledge about cortical sensory integration processes.

\section{Key Studies}

In the following, we highlight one key study in the area of walking and balance. Those studies are of high practical relevance and cannot be performed in an fMRI since motor imagery is suggested not to be a satisfactory indicative of brain activation during motor execution. ${ }^{257}$

\subsection{Walking}

The usage of a smartphone during walking causes serious injuries. ${ }^{258,259}$ Hence, the understanding and the analysis of underlying motor control processes of walking while texting on a smartphone seems to be of high practical relevance. ${ }^{260}$ The investigation of smartphone usage while recording the kinematics of gait is not possible in an fMRI-scanner but could be conducted with fNIRS. In the study of Takeuchi et al., ${ }^{89}$ the influence of using a smartphone while walking was investigated in healthy old and young adults. Takeuchi et al. ${ }^{89}$ observed that in young adults, the activation magnitude of left PFC is associated with dual-task cost (change between single- and dual-task performances) of gait acceleration and right PFC is related to the dual-task cost of the conducted cognitive smartphone task. In contrast, in the older adults middle PFC was associated with dual-task costs of step time and the activation of the left PFC is associated with dual-task costs of gait acceleration. ${ }^{89}$ Furthermore, younger adults have lower dual-task costs in kinematic parameters. ${ }^{89}$ In sum, these results point toward the effective lateralization in young adults, while in older adults more resources are needed to maintain gait performance which is in accordance with the theories of hemispheric asymmetry reduction $^{261}$ and compensational recruitment. ${ }^{262}$

\subsection{Postural Tasks}

While fMRT is sensitive to motion artifacts, ${ }^{18-21}$ the simultaneous recording of brain activity and the quantification of kinematic parameters of gross motor skills (e.g., dynamic whole-body balance task) are impossible. Remarkably, it is assumed that to increase our knowledge about neuromotor control processes, the simultaneous assessment of brain activity and kinematic parameters is necessary. ${ }^{263}$ Furthermore, gross motor skills are, for example, an essential part of rehabilitative interventions (e.g., balancing on wobble board ${ }^{264-266}$ ). The study of Herold et al. ${ }^{100}$ used fNIRS to investigate the contribution of motor areas in online neuromotor control of balance performance on a wobble board and recorded simultaneous sway parameters via an inertial sensor. They observed (1) a pronounced activation of PrG, PoG, and SMA during balancing and (2) a strong negative correlation between the magnitude of SMA activation and sway in mediolateral direction during balancing. ${ }^{100}$ The results of Herold et al. ${ }^{100}$ allow a deeper understanding of the role of the SMA in online neuromotor control of balance movements and may be helpful to design tailored intervention programs or to monitor the intervention progress.

\section{Conclusion}

In sum, neuroimaging with the fNIRS technology seems to be a promising tool to shed light on the functioning of cortical areas in motor control. However, the absence of standardized study protocols limits the comparability among studies. Based on our findings, we deduce recommendations and potential future directions, which are shown in Table 2. Hopefully, those recommendations will lay foundations to improve the study protocols and data processing of fNIRS methodology encouraging further research to extend our existing knowledge about neuromotor control processes. This increase in knowledge might be helpful to develop tailored rehabilitation programs for clinical settings in, e.g., orthopedics and neurology. ${ }^{42}$ Furthermore, combining the information we can derive from fNIRS signals with kinematic parameters which are risk factors for falls ${ }^{132,267}$ or for 
Table 2 Recommendations for future fNIRS studies.

Recommendations:

- Report all technical configuration details (source-detector separation, wavelengths, sampling frequency, number of measurement channels, DPF values with selection process, etc.) and design-related details (e.g., duration of task and rest phases).

- Optode placement should be based on the 10 to 20 EEG system.

- Additional measures (e.g., heart rate, blood pressure, respiration, skin conductance, etc.) should be used to monitor systematic changes.

- In order to process data, the use of bandpass filters and wavelet filters is recommended.

- DPF values should be calculated depending on age and cortex region or directly quantified via frequency- or time-domain NIRS.

- Physiological cofounders (e.g., scalp blood flow) should be reduced with the aid of PCA/ICA analyses or the usage of short separation channels.

- Baseline correction or baseline normalization should be applied.

- Averaging across channels of a ROI and trials seems to be favorable.

- The relative changes of both, oxyHb and deoxyHb, should be reported and used in the statistical analysis.

cognitive decline ${ }^{268}$ could perhaps support a more sensitive and effective early detection of persons with a high likelihood for falls or with a high risk to develop cognitive diseases. This, in turn, may allow an early onset of therapeutic interventions, an effective monitoring of intervention programs and it would support the decision making in health care units. Those potential applications could be beneficial for patients and the resources of the health care system.

\section{Appendix}

For further information about search strategy, cohort characteristics, study protocols, number of fNIRS channels, used wavelengths and sampling frequencies in the reviewed studies, we provide supplemental content which is available in Ref. 52, or can be requested by e-mail from the corresponding author.

\section{Disclosures}

The authors declare no conflict of interest.

\section{References}

1. D. Hamacher et al., "Brain activity during walking: a systematic review," Neurosci. Biobehav. Rev. 57, 310-327 (2015).

2. R. Holtzer et al., "Neuroimaging of mobility in aging: a targeted review," J. Gerontol. Ser. A: Biol. Sci. Med. Sci. 69(11), 1375-1388 (2014).

3. J. V. Jacobs and F. B. Horak, "Cortical control of postural responses," J. Neural Transm. 114(10), 1339-1348 (2007).

4. K. Takakusaki, "Neurophysiology of gait: from the spinal cord to the frontal lobe," Mov. Disord.: Off. J. Mov. Disord. Soc. 28(11), 1483-1491 (2013).

5. A. L. Rosso et al., "Higher step length variability indicates lower gray matter integrity of selected regions in older adults," Gait Posture 40(1), 225-230 (2014).

6. O. Beauchet et al., "Higher gait variability is associated with decreased parietal gray matter volume among healthy older adults," Brain Topogr. 27(2), 293-295 (2014).

7. Q. Tian et al., "Lower gray matter integrity is associated with greater lap time variation in high-functioning older adults," Exp. Gerontol. 77, 46-51 (2016).

8. C. Rosano et al., "Slower gait, slower information processing and smaller prefrontal area in older adults," Age Ageing 41(1), 58-64 (2011).
9. M. L. Callisaya et al., "Global and regional associations of smaller cerebral gray and white matter volumes with gait in older people," PLoS One 9(1), e84909 (2014).

10. M. P. Boisgontier et al., "Whole-brain grey matter density predicts balance stability irrespective of age and protects older adults from falling," Gait Posture 45, 143-150 (2016).

11. M. Taubert et al., "Dynamic properties of human brain structure: learning-related changes in cortical areas and associated fiber connections," J. Neurosci. 30(35), 11670-11677 (2010).

12. M. Taubert et al., "Rapid and specific gray matter changes in M1 induced by balance training," Neurolmage 133, 399-407 (2016).

13. M. Taubert, A. Villringer, and P. Ragert, "Learning-related gray and white matter changes in humans: an update," Neuroscientist 18(4), 320-325 (2012).

14. N. Raz et al., "Regional brain changes in aging healthy adults: general trends, individual differences and modifiers," Cereb. Cortex 15(11), 1676-1689 (2005).

15. N. Raz et al., "Selective aging of the human cerebral cortex observed in vivo: differential vulnerability of the prefrontal gray matter," Cereb. Cortex 7(3), 268-282 (1997).

16. R. D. Seidler et al., "Motor control and aging: links to age-related brain structural, functional, and biochemical effects," Neurosci. Biobehav. Rev. 34(5), 721-733 (2010).

17. S. Perrey, "Possibilities for examining the neural control of gait in humans with fNIRS," Front. Physiol. 5, 282 (2014).

18. S. Cutini and S. Brigadoi, "Unleashing the future potential of functional near-infrared spectroscopy in brain sciences," J. Neurosci. Methods 232, 152-156 (2014).

19. S. C. Bunce et al., "Functional near-infrared spectroscopy," IEEE Eng. Med. Biol. Mag. 25(4), 54-62 (2006).

20. J. Saliba et al., "Functional near-infrared spectroscopy for neuroimaging in cochlear implant recipients," Hear. Res. 338, 64-75 (2016).

21. S. Cutini, S. Moro, and S. Bisconti, "Review: functional near infrared optical imaging in cognitive neuroscience: an introductory review," J. Near Infrared Spectrosc. 20(1), 75 (2012).

22. S. Lloyd-Fox, A. Blasi, and C. E. Elwell, "Illuminating the developing brain: the past, present and future of functional near infrared spectroscopy," Neurosci. Biobehav. Rev. 34(3), 269-284 (2010).

23. J. Thompson, W. Sebastianelli, and S. Slobounov, "EEG and postural correlates of mild traumatic brain injury in athletes," Neurosci. Lett. 377(3), 158-163 (2005).

24. M. Smith, "Shedding light on the adult brain: a review of the clinical applications of near-infrared spectroscopy," Philos. Trans. R. Soc. A 369(1955), 4452-4469 (2011).

25. D. R. Leff et al., "Assessment of the cerebral cortex during motor task behaviours in adults: a systematic review of functional near infrared spectroscopy (fNIRS) studies," NeuroImage 54(4), 2922-2936 (2011). 
26. A. Villringer and B. Chance, "Non-invasive optical spectroscopy and imaging of human brain function," Trends Neurosci. 20(10), 435-442 (1997).

27. S. Perrey, "Non-invasive NIR spectroscopy of human brain function during exercise," Methods 45(4), 289-299 (2008).

28. C. Huneau, H. Benali, and H. Chabriat, "Investigating human neurovascular coupling using functional neuroimaging: a critical review of dynamic models," Front. Neurosci. 9, e1002435 (2015).

29. L.-D. Liao et al., "Neurovascular coupling: in vivo optical techniques for functional brain imaging," BioMed. Eng. OnLine 12(1), 38 (2013).

30. H. Obrig and A. Villringer, "Beyond the visible imaging the human brain with light," J. Cereb. Blood Flow Metab. 23(1), 1-18 (2003).

31. H. Obrig et al., "Near-infrared spectroscopy. Does it function in functional activation studies of the adult brain?" Int. J. Psychophysiol. 35(2-3), 125-142 (2000).

32. M. Izzetoglu et al., "Functional brain imaging using near-infrared technology," IEEE Eng. Med. Biol. Mag. 26(4), 38-46 (2007).

33. F. Scholkmann et al., "A review on continuous wave functional nearinfrared spectroscopy and imaging instrumentation and methodology," NeuroImage 85, 6-27 (2014).

34. J. León-Carrión and U. León-Domínguez, "Functional near-infrared spectroscopy (fNIRS): principles and neuroscientific applications," in Neuroimaging Methods, P. Bright, Ed., pp. 48-74, INTECH Open Access Publisher, Rijeka, Croatia (2017).

35. M. Ferrari and V. Quaresima, "A brief review on the history of human functional near-infrared spectroscopy (fNIRS) development and fields of application," NeuroImage 63(2), 921-935 (2012).

36. V. Quaresima, S. Bisconti, and M. Ferrari, "A brief review on the use of functional near-infrared spectroscopy (fNIRS) for language imaging studies in human newborns and adults," Brain Lang. 121(2), 79-89 (2012).

37. Y. Minagawa-Kawai et al., "Optical imaging of infants' neurocognitive development: recent advances and perspectives," Dev. Neurobiol. 68(6), 712-728 (2008).

38. H. Shibasaki, "Human brain mapping: hemodynamic response and electrophysiology," Clin. Neurophysiol. 119(4), 731-743 (2008).

39. T. Wilcox and M. Biondi, "fNIRS in the developmental sciences," Wiley Interdiscip. Rev. Cognit. Sci. 6(3), 263-283 (2015).

40. J. Gervain et al., "Near-infrared spectroscopy: a report from the McDonnell infant methodology consortium," Dev. Cognit. Neurosci. 1(1), 22-46 (2011).

41. S. K. Piper et al., "A wearable multi-channel fNIRS system for brain imaging in freely moving subjects," NeuroImage 85, 64-71 (2014).

42. H. Obrig, "NIRS in clinical neurology-a "promising' tool?" NeuroImage 85, 535-546 (2014).

43. M. Mihara and I. Miyai, "Review of functional near-infrared spectroscopy in neurorehabilitation," Neurophotonics 3(3), 031414 (2016).

44. V. Gramigna et al., "Near-infrared spectroscopy in gait disorders. Is it time to begin?," Neurorehabil. Neural Repair 31(5), 402-412 (2017).

45. I. Tachtsidis and F. Scholkmann, "False positives and false negatives in functional near-infrared spectroscopy: issues, challenges, and the way forward," Neurophotonics 3(3), 030401 (2016).

46. E. Tanaka, S. Saegusa, and L. Yuge, "Development of a whole body motion support type mobile suit and evaluation of cerebral activity corresponding to the cortical motor areas," J. Adv. Mech. Des. Syst. Manuf. 7(1), 82-94 (2013).

47. T. Sukal-Moulton et al., "Functional near infrared spectroscopy of the sensory and motor brain regions with simultaneous kinematic and EMG monitoring during motor tasks," J. Visualized Exp. 5 (94), 52391 (2014).

48. P. Pinti et al., "Using fiberless, wearable fNIRS to monitor brain activity in real-world cognitive tasks," J. Visualized Exp. 2(106), e53336 (2015).

49. S. Suzuki, F. Harashima, and K. Furuta, "Human control law and brain activity of voluntary motion by utilizing a balancing task with an inverted pendulum," Adv. Hum.-Comput. Interact. 2010(2), 1-16 (2010).

50. S. Imaoka and E. Matubara, "Postural adjustment mechanisms of cerebrovascular disease patients: a comparison with healthy adults using near-infrared spectroscopy," Rigakuryoho Kagaku 30(6), 981985 (2015).

51. I. Naitou et al., "The dynamics of blood oxygen in the brain of healthy young adults in the performance of various walking styles," Rigakuryoho Kagaku 28(4), 435-440 (2013).
52. F. Herold et al., Appendix to "Functional near-infrared spectroscopy in movement science: a systematic review on cortical activity in postural and walking tasks," https://www.researchgate.net/profile/Fabian_Herold (2017).

53. T. Harada et al., "Gait capacity affects cortical activation patterns related to speed control in the elderly," Exp. Brain Res. 193(3), 445-454 (2009).

54. K. L. Koenraadt et al., "Cortical control of normal gait and precision stepping: an fNIRS study," NeuroImage 85, 415-422 (2014).

55. M. J. Kurz, T. W. Wilson, and D. J. Arpin, "Stride-time variability and sensorimotor cortical activation during walking," NeuroImage 59(2), 1602-1607 (2012).

56. M. J. Kurz, T. W. Wilson, and D. J. Arpin, "An fNIRS exploratory investigation of the cortical activity during gait in children with spastic diplegic cerebral palsy," Brain Dev. 36(10), 870-877 (2014).

57. D. Meester et al., "Associations between prefrontal cortex activation and H-reflex modulation during dual task gait," Front. Hum. Neurosci. 8, 78 (2014).

58. M. Mihara et al., "Sustained prefrontal activation during ataxic gait: a compensatory mechanism for ataxic stroke?" NeuroImage 37(4), 1338-1345 (2007).

59. I. Miyai et al., "Effect of body weight support on cortical activation during gait in patients with stroke," Exp. Brain Res. 169(1), 85-91 (2006).

60. I. Miyai et al., "Longitudinal optical imaging study for locomotor recovery after stroke," Stroke 34(12), 2866-2870 (2003).

61. I. Miyai et al., "Premotor cortex is involved in restoration of gait in stroke," Ann. Neurol. 52(2), 188-194 (2002).

62. M. Suzuki et al., "Prefrontal and premotor cortices are involved in adapting walking and running speed on the treadmill: an optical imaging study," NeuroImage 23(3), 1020-1026 (2004).

63. S. A. Fraser et al., "Comparable cerebral oxygenation patterns in younger and older adults during dual-task walking with increasing load," Front. Aging Neurosci. 8, 240 (2016).

64. F. G. Metzger et al., "Functional brain imaging of walking while talking-an fNIRS study," Neuroscience 343, 85-93 (2017).

65. E. Al-Yahya et al., "Prefrontal cortex activation while walking under dual-task conditions in stroke: a multimodal imaging study," Neurorehabil. Neural Repair 30(6), 591-599 (2016).

66. D. J. Clark et al., "Enhanced somatosensory feedback reduces prefrontal cortical activity during walking in older adults," J. Gerontol. Ser. A: Biol. Sci. Med. Sci. 69(11), 1422-1428 (2014).

67. M. Suzuki et al., "Activities in the frontal cortex and gait performance are modulated by preparation: an fNIRS study," Neurolmage 39(2), 600-607 (2008).

68. P. Eggenberger et al., "Exergame and balance training modulate prefrontal brain activity during walking and enhance executive function in older adults," Front. Aging Neurosci. 8, 66 (2016).

69. H. Atsumori et al., "Noninvasive imaging of prefrontal activation during attention-demanding tasks performed while walking using a wearable optical topography system," J. Biomed. Opt. 15(4), 046002 (2010).

70. P. Caliandro et al., "Prefrontal cortex controls human balance during overground ataxic gait," Restor. Neurol. Neurosci. 30(5), 397-405 (2012).

71. P. Caliandro et al., "Prefrontal cortex as a compensatory network in ataxic gait: a correlation study between cortical activity and gait parameters," Restor. Neurol. Neurosci. 33(2), 177-187 (2015).

72. T. Doi et al., "Brain activation during dual-task walking and executive function among older adults with mild cognitive impairment: a fNIRS study," Aging Clin. Exp. Res. 25(5), 539-544 (2013).

73. R. Holtzer et al., "fNIRS study of walking and walking while talking in young and old individuals," J. Gerontol. Ser. A: Biol. Sci. Med. Sci. 66A(8), 879-887 (2011).

74. R. Holtzer et al., "Online fronto-cortical control of simple and attentiondemanding locomotion in humans," Neurolmage 112, 152-159 (2015).

75. R. Holtzer et al., "Neurological gait abnormalities moderate the functional brain signature of the posture first hypothesis," Brain Topogr. 29(2), 334-343 (2016).

76. C.-F. Lu et al., "Maintaining gait performance by cortical activation during dual-task interference: a functional near-infrared spectroscopy study," PLoS One 10(6), e0129390 (2015). 
77. A. Mirelman et al., "Increased frontal brain activation during walking while dual tasking: an fNIRS study in healthy young adults," J. Neuroeng. Rehabil. 11, 85 (2014).

78. H. Saitou et al., "Cerebral blood volume and oxygenation among poststroke hemiplegic patients: effects of 13 rehabilitation tasks measured by near-infrared spectroscopy," Arch. Phys. Med. Rehabil. 81(10), $1348-1356$ (2000).

79. M.-I. B. Lin and K.-H. Lin, "Walking while performing working memory tasks changes the prefrontal cortex hemodynamic activations and gait kinematics," Front. Behav. Neurosci. 10, 92 (2016).

80. I. Maidan et al., "The role of the frontal lobe in complex walking among patients with Parkinson's disease and healthy older adults: an fNIRS study," Neurorehabil. Neural Repair 30(10), 963-971 (2016).

81. F. Nieuwhof et al., "Measuring prefrontal cortical activity during dual task walking in patients with Parkinson's disease. Feasibility of using a new portable fNIRS device," Pilot Feasibility Stud. 2(1), 918719 (2016).

82. M. E. Hernandez et al., "Brain activation changes during locomotion in middle-aged to older adults with multiple sclerosis," J. Neurol. Sci. 370, 277-283 (2016).

83. J. Verghese et al., "Brain activation in high-functioning older adults and falls: prospective cohort study," Neurology 88(2), 191-197 (2016).

84. R. Holtzer et al., "Stress and gender effects on prefrontal cortex oxygenation levels assessed during single and dual-task walking conditions," Eur. J. Neurosci. 45(5), 660-670 (2016).

85. R. Holtzer et al., "Interactions of subjective and objective measures of fatigue defined in the context of brain control of locomotion," J. Gerontol. Ser. A Biol. Sci. Med. Sci. 72(3), 417-423 (2017).

86. D. J. Clark et al., "Utilization of central nervous system resources for preparation and performance of complex walking tasks in older adults," Front. Aging Neurosci. 6, 217 (2014).

87. O. Osofundiya et al., "Obesity-specific neural cost of maintaining gait performance under complex conditions in community-dwelling older adults," Clin. Biomech. 35, 42-48 (2016).

88. R. McKendrick et al., "Prefrontal hemodynamics of physical activity and environmental complexity during cognitive work," Hum. Factors 59(1), 147-162 (2017).

89. N. Takeuchi et al., "Parallel processing of cognitive and physical demands in left and right prefrontal cortices during smartphone use while walking," BMC Neurosci. 17(1), 9 (2016).

90. I. Maidan et al., "Changes in oxygenated hemoglobin link freezing of gait to frontal activation in patients with Parkinson disease: an fNIRS study of transient motor-cognitive failures," J. Neurol. 262(4), 899908 (2015).

91. T. Huppert et al., "Measurement of brain activation during an upright stepping reaction task using functional near-infrared spectroscopy," Hum. Brain Mapp. 34(11), 2817-2828 (2013).

92. H. Karim et al., "Functional brain imaging of multi-sensory vestibular processing during computerized dynamic posturography using nearinfrared spectroscopy," NeuroImage 74, 318-325 (2013).

93. J. R. Mahoney et al., "The role of prefrontal cortex during postural control in Parkinsonian syndromes a functional near-infrared spectroscopy study," Brain Res. 1633, 126-138 (2016).

94. M. Mihara et al., "Role of the prefrontal cortex in human balance control," NeuroImage 43(2), 329-336 (2008).

95. M. Mihara et al., "Cortical control of postural balance in patients with hemiplegic stroke," NeuroReport 23(5), 314-319 (2012).

96. H. Takakura et al., "Cerebral hemodynamic responses during dynamic posturography. Analysis with a multichannel near-infrared spectroscopy system," Front. Hum. Neurosci. 9, 620 (2015).

97. H. Karim et al., "Functional near-infrared spectroscopy (fNIRS) of brain function during active balancing using a video game system," Gait Posture 35(3), 367-372 (2012).

98. S. Basso Moro et al., "A semi-immersive virtual reality incremental swing balance task activates prefrontal cortex: a functional near-infrared spectroscopy study," NeuroImage 85(Pt 1), 451-460 (2014).

99. M. Ferrari et al., "Prefrontal cortex activated bilaterally by a tilt board balance task: a functional near-infrared spectroscopy study in a semiimmersive virtual reality environment," Brain Topogr. 27(3), 353-365 (2014).

100. F. Herold et al., "Cortical activation during balancing on a balance board," Hum. Mov. Sci. 51, 51-58 (2017).
101. H. Fujita et al., "Role of the frontal cortex in standing postural sway tasks while dual-tasking: a functional near-infrared spectroscopy study examining working memory capacity," BioMed. Res. Int. 2016(5), 1-10 (2016).

102. H. Fujimoto et al., "Cortical changes underlying balance recovery in patients with hemiplegic stroke," NeuroImage 85, 547-554 (2014).

103. C.-C. Lin et al., "Functional near-infrared spectroscopy (fNIRS) brain imaging of multi-sensory integration during computerized dynamic posturography in middle-aged and older adults," Exp. Brain Res. 235(4), 1247-1256 (2017).

104. B. Wang et al., "Posture-related changes in brain functional connectivity as assessed by wavelet phase coherence of NIRS signals in elderly subjects," Behav. Brain Res. 312, 238-245 (2016).

105. R. Beurskens et al., "Age-related changes in prefrontal activity during walking in dual-task situations: a fNIRS study," Int. J. Psychophysiol. 92(3), 122-128 (2014).

106. H. Y. Kim et al., "Best facilitated cortical activation during different stepping, treadmill, and robot-assisted walking training paradigms and speeds: a functional near-infrared spectroscopy neuroimaging study," NeuroRehabilitation 38(2), 171-178 (2016).

107. I. Miyai et al., "Cortical mapping of gait in humans: a near-infrared spectroscopic topography study," NeuroImage 14(5), 1186-1192 (2001).

108. I. Helmich, A. Berger, and H. Lausberg, "Neural control of posture in individuals with persisting postconcussion symptoms," Med. Sci. Sports Exercise 48(12), 2362-2369 (2016).

109. G. Strangman, M. A. Franceschini, and D. A. Boas, "Factors affecting the accuracy of near-infrared spectroscopy concentration calculations for focal changes in oxygenation parameters," NeuroImage 18(4), 865-879 (2003).

110. F. Scholkmann and M. Wolf, "General equation for the differential pathlength factor of the frontal human head depending on wavelength and age," J. Biomed. Opt. 18(10), 105004 (2013).

111. T. J. Huppert et al., "HomER: a review of time-series analysis methods for near-infrared spectroscopy of the brain," Appl. Opt. 48(10), D280 (2009).

112. R. E. Vanderwert and C. A. Nelson, "The use of near-infrared spectroscopy in the study of typical and atypical development," NeuroImage 85(Pt 1), 264-271 (2014).

113. M. A. Killingsworth and D. T. Gilbert, "A wandering mind is an unhappy mind," Science 330(6006), 932-932 (2010).

114. G. Berthie et al., "The restless mind while driving: drivers' thoughts behind the wheel," Accid. Anal. Prev. 76, 159-165 (2015).

115. C. Galera et al., "Mind wandering and driving: responsibility casecontrol study," BMJ 345, e8105 (2012).

116. C. T. Lin et al., "Mind-wandering tends to occur under low perceptual demands during driving," Sci. Rep. 6, 21353 (2016).

117. M. Spronken et al., "Temporal focus, temporal distance, and mindwandering valence: results from an experience sampling and an experimental study," Conscious Cognit. 41, 104-118 (2016).

118. M. F. Mason et al., "Wandering minds: the default network and stimulus-independent thought," Science 315(5810), 393-395 (2007).

119. K. C. Fox et al., "Dreaming as mind wandering: evidence from functional neuroimaging and first-person content reports," Front. Hum. Neurosci. 7, 412 (2013).

120. G. Durantin, F. Dehais, and A. Delorme, "Characterization of mind wandering using fNIRS," Front. Syst. Neurosci. 9, 98 (2015).

121. P. Corbeil et al., "Perturbation of the postural control system induced by muscular fatigue," Gait Posture 18(2), 92-100 (2003).

122. P. A. Gribble and J. Hertel, "Effect of lower-extremity muscle fatigue on postural control," Arch. Phys. Med. Rehabil. 85(4), 589-592 (2004).

123. J. L. Helbostad et al., "Consequences of lower extremity and trunk muscle fatigue on balance and functional tasks in older people: a systematic literature review," BMC Geriatr. 10, 56 (2010).

124. T. Paillard, "Effects of general and local fatigue on postural control: a review," Neurosci. Biobehav. Rev. 36(1), 162-176 (2012).

125. M. Salavati et al., "Changes in postural stability with fatigue of lower extremity frontal and sagittal plane movers," Gait Posture 26(2), 214-218 (2007).

126. J. A. Yaggie and S. J. McGregor, "Effects of isokinetic ankle fatigue on the maintenance of balance and postural limits," Arch. Phys. Med. Rehabil. 83(2), 224-228 (2002). 
127. A. E. Shortz et al., "The effect of cognitive fatigue on prefrontal cortex correlates of neuromuscular fatigue in older women," J. NeuroEng. Rehabil. 12, 115 (2015).

128. M. L. Latash, J. P. Scholz, and G. Schöner, "Motor control strategies revealed in the structure of motor variability," Exercise Sport Sci. Rev. 30(1), 26-31 (2002).

129. R. T. Harbourne and N. Stergiou, "Movement variability and the use of nonlinear tools: principles to guide physical therapist practice," Phys. Ther. 89(3), 267-282 (2009).

130. N. Stergiou, R. Harbourne, and J. Cavanaugh, "Optimal movement variability: a new theoretical perspective for neurologic physical therapy," J. Neurol. Phys. Ther. 30(3), 120-129 (2006).

131. N. Stergiou and L. M. Decker, "Human movement variability, nonlinear dynamics, and pathology: is there a connection?" Hum. Mov. Sci. 30(5), 869-888 (2011).

132. D. Hamacher et al., "Kinematic measures for assessing gait stability in elderly individuals: a systematic review," J. R. Soc. Interface 8(65), 1682-1698 (2011).

133. N. König et al., "Revealing the quality of movement: a meta-analysis review to quantify the thresholds to pathological variability during standing and walking," Neurosci. Biobehav. Rev. 68, 111-119 (2016).

134. J. H. Hollman et al., "Number of strides required for reliable measurements of pace, rhythm and variability parameters of gait during normal and dual task walking in older individuals," Gait Posture 32(1), 23-28 (2010).

135. F. Riva, M. C. Bisi, and R. Stagni, "Gait variability and stability measures: minimum number of strides and within-session reliability," Comput. Biol. Med. 50, 9-13 (2014).

136. A. F. Cannestra et al., "Refractory periods observed by intrinsic signal and fluorescent dye imaging," J. Neurophysiol. 80(3), 1522-1532 (1998).

137. A. Pellicer and M. del Carmen Bravo, "Near-infrared spectroscopy: a methodology-focused review," Semin. Fetal Neonat. Med. 16(1), 42-49 (2011).

138. M. Ferrari, L. Mottola, and V. Quaresima, "Principles, techniques, and limitations of near infrared spectroscopy," Can. J. Appl. Physiol. 29(4), 463-487 (2004).

139. F. Orihuela-Espina et al., "Quality control and assurance in functional near infrared spectroscopy (fNIRS) experimentation," Phys. Med. Biol. 55(13), 3701-3724 (2010).

140. D. A. Benaron et al., "Transcranial optical path length in infants by near-infrared phase-shift spectroscopy," J. Clin. Monit. Comput. 11(2), 109-117 (1995).

141. S. Brigadoi and R. J. Cooper, "How short is short? Optimum sourcedetector distance for short-separation channels in functional nearinfrared spectroscopy," Neurophotonics 2(2), 025005 (2015).

142. G. E. Strangman, Q. Zhang, and Z. Li, "Scalp and skull influence on near infrared photon propagation in the Colin27 brain template," NeuroImage 85(Pt 1), 136-149 (2014).

143. S. Wang et al., "Effects of spatial variation of skull and cerebrospinal fluid layers on optical mapping of brain activities," Opt. Rev. 17(4), $410-420$ (2010).

144. K. L. Perdue, Q. Fang, and S. G. Diamond, "Quantitative assessment of diffuse optical tomography sensitivity to the cerebral cortex using a whole-head probe," Phys. Med. Biol. 57(10), 2857-2872 (2012).

145. T. Funane et al., "Quantitative evaluation of deep and shallow tissue layers' contribution to fNIRS signal using multi-distance optodes and independent component analysis," Neurolmage 85(Pt 1), 150-165 (2014).

146. T. Funane et al., "Greater contribution of cerebral than extracerebral hemodynamics to near-infrared spectroscopy signals for functional activation and resting-state connectivity in infants," Neurophotonics 1(2), 025003 (2014).

147. A. V. Patil et al., "Experimental investigation of NIRS spatial sensitivity," Biomed. Opt. Express 2(6), 1478-1493 (2011).

148. S. Gunadi et al., "Spatial sensitivity and penetration depth of three cerebral oxygenation monitors," Biomed. Opt. Express 5(9), 2896-2912 (2014).

149. V. Toronov et al., "Near-infrared study of fluctuations in cerebral hemodynamics during rest and motor stimulation: temporal analysis and spatial mapping," Med. Phys. 27(4), 801-815 (2000).
150. S. Kohri et al., "Quantitative evaluation of the relative contribution ratio of cerebral tissue to near-infrared signals in the adult human head: a preliminary study," Physiol. Meas. 23(2), 301-312 (2002).

151. D. P. Carmody et al., "A quantitative measure of myelination development in infants, using MR images," Neuroradiology 46(9), 781-786 (2004).

152. A. Giorgio et al., "Age-related changes in grey and white matter structure throughout adulthood," NeuroImage 51(3), 943-951 (2010).

153. X. Wang et al., "Probabilistic MRI brain anatomical atlases based on 1,000 Chinese subjects," PLoS One 8(1), e50939 (2013).

154. K. L. Leenders et al., "Cerebral blood flow, blood volume and oxygen utilization: normal values and effect of age," Brain: J. Neurol. 113(Pt 1), 27-47 (1990).

155. A. Duncan et al., "Measurement of cranial optical path length as a function of age using phase resolved near infrared spectroscopy," Pediatr. Res. 39(5), 889-894 (1996).

156. P. van der Zee et al., "Experimentally measured optical pathlengths for the adult head, calf and forearm and the head of the newborn infant as a function of inter optode spacing," in Oxygen Transport to Tissue XIII, T. K. Goldstick, M. McCabe, and D. J. Maguire, Eds., Vol. 316, pp. 143-153, Springer, Boston, Massachusetts (1992).

157. M. Essenpreis et al., "Spectral dependence of temporal point spread functions in human tissues," Appl. Opt. 32(4), 418-425 (1993).

158. H. Zhao et al., "Maps of optical differential pathlength factor of human adult forehead, somatosensory motor and occipital regions at multi-wavelengths in NIR," Phys. Med. Biol. 47(12), 2075-2093 (2002).

159. Y. Hoshi, "Functional near-infrared spectroscopy: potential and limitations in neuroimaging studies," Int. Rev. Neurobiol 66(5), 237-266 (2005).

160. Y. Hoshi, "Functional near-infrared spectroscopy: current status and future prospects," J. Biomed. Opt. 12(6), 062106 (2007).

161. A. Maki et al., "Spatial and temporal analysis of human motor activity using noninvasive NIR topography," Med. Phys. 22(12), 1997-2005 (1995).

162. M. M. Tisdall et al., "The effect on cerebral tissue oxygenation index of changes in the concentrations of inspired oxygen and end-tidal carbon dioxide in healthy adult volunteers," Anesth. Analg. 109(3), 906-913 (2009).

163. M. Wolf, M. Ferrari, and V. Quaresima, "Progress of near-infrared spectroscopy and topography for brain and muscle clinical applications," J. Biomed. Opt. 12(6), 062104 (2007).

164. V. Quaresima and M. Ferrari, "Muscle oxygenation by near-infraredbased tissue oximeters," J. Appl. Physiol. 107(1), 371 (2009).

165. P. Ekkekakis, "Illuminating the black box: investigating prefrontal cortical hemodynamics during exercise with near-infrared spectroscopy," J. Sport Exercise Psychol. 31(4), 505-553 (2009).

166. M. L. Schroeter et al., "Age dependency of the hemodynamic response as measured by functional near-infrared spectroscopy," NeuroImage 19(3), 555-564 (2003).

167. S. Brigadoi et al., "Motion artifacts in functional near-infrared spectroscopy: a comparison of motion correction techniques applied to real cognitive data," NeuroImage 85, 181-191 (2014).

168. R. J. Cooper et al., "A systematic comparison of motion artifact correction techniques for functional near-infrared spectroscopy," Front. Neurosci. 6, 147 (2012).

169. Y. Zhang et al., "Eigenvector-based spatial filtering for reduction of physiological interference in diffuse optical imaging," J. Biomed. Opt. 10(1), 011014 (2005).

170. H. Santosa et al., "Noise reduction in functional near-infrared spectroscopy signals by independent component analysis," Rev. Sci. Instrum. 84(7), 073106 (2013).

171. F. C. Robertson, T. S. Douglas, and E. M. Meintjes, "Motion artifact removal for functional near infrared spectroscopy: a comparison of methods," IEEE Trans. BioMed. Eng. 57(6), 1377-1387 (2010).

172. H. Tanaka, T. Katura, and H. Sato, "Task-related oxygenation and cerebral blood volume changes estimated from NIRS signals in motor and cognitive tasks," NeuroImage 94, 107-119 (2014).

173. H. Tanaka, T. Katura, and H. Sato, "Task-related component analysis for functional neuroimaging and application to near-infrared spectroscopy data," NeuroImage 64, 308-327 (2013). 
174. M. A. Yucel et al., "Target principal component analysis: a new motion artefact correction approach for near-infrared spectroscopy," J. Innovative Opt. Health Sci. 7(2), 1350066 (2014).

175. X. Cui, S. Bray, and A. L. Reiss, "Functional near infrared spectroscopy (NIRS) signal improvement based on negative correlation between oxygenated and deoxygenated hemoglobin dynamics," NeuroImage 49(4), 3039-3046 (2010).

176. B. Molavi and G. A. Dumont, "Wavelet-based motion artifact removal for functional near-infrared spectroscopy," Physiol. Meas. 33(2), 259270 (2012).

177. K. E. Jang et al., "Wavelet minimum description length detrending for near-infrared spectroscopy," J. Biomed. Opt. 14(3), 034004 (2009).

178. A. M. Chiarelli et al., "A kurtosis-based wavelet algorithm for motion artifact correction of fNIRS data," NeuroImage 112, 128-137 (2015).

179. H. Sato et al., "Wavelet analysis for detecting body-movement artifacts in optical topography signals," NeuroImage 33(2), 580-587 (2006).

180. J. W. Barker, A. Aarabi, and T. J. Huppert, "Autoregressive model based algorithm for correcting motion and serially correlated errors in fNIRS," Biomed. Opt. Express 4(8), 1366-1379 (2013).

181. M. Izzetoglu et al., "Motion artifact cancellation in NIR spectroscopy using discrete Kalman filtering," BioMed. Eng. OnLine 9(1), 16 (2010).

182. M. Izzetoglu et al., "Motion artifact cancellation in NIR spectroscopy using Wiener filtering," IEEE Trans. Biomed. Eng. 52(5), 934-938 (2005).

183. T. Nozawa and T. Kondo, "A comparison of artifact reduction methods for real-time analysis of fNIRS data," in Human Interface and the Management of Information: Information and Interaction, D. Hutchison, Ed., Vol. 5618, pp. 413-422, Springer, Berlin, Heidelberg (2009).

184. F. Scholkmann et al., "How to detect and reduce movement artifacts in near-infrared imaging using moving standard deviation and spline interpolation," Physiol. Meas. 31(5), 649-662 (2010).

185. F. Scholkmann et al., "End-tidal $\mathrm{CO}_{2}$ : an important parameter for a correct interpretation in functional brain studies using speech tasks," Neurolmage 66, 71-79 (2013).

186. F. Scholkmann, M. Wolf, and U. Wolf, "The effect of inner speech on arterial $\mathrm{CO}_{2}$ and cerebral hemodynamics and oxygenation: a functional NIRS study," Adv. Exp. Med. Biol. 789, 81-87 (2013).

187. D. A. Boas, A. M. Dale, and M. A. Franceschini, "Diffuse optical imaging of brain activation: approaches to optimizing image sensitivity, resolution, and accuracy," Neurolmage 23(Suppl. 1), S275-S288 (2004).

188. M. Caldwell et al., "Modelling confounding effects from extracerebral contamination and systemic factors on functional near-infrared spectroscopy," NeuroImage 143, 91-105 (2016).

189. G. Gratton and P. M. Corballis, "Removing the heart from the brain: compensation for the pulse artifact in the photon migration signal," Psychophysiology 32(3), 292-299 (1995).

190. G. Morren et al., "Detection of fast neuronal signals in the motor cortex from functional near infrared spectroscopy measurements using independent component analysis," Med. Biol. Eng. Comput. 42(1), 92-99 (2004).

191. L. Holper, F. Scholkmann, and M. Wolf, "The relationship between sympathetic nervous activity and cerebral hemodynamics and oxygenation: a study using skin conductance measurement and functional near-infrared spectroscopy," Behav. Brain Res. 270, 95-107 (2014).

192. S. Tak and J. C. Ye, "Statistical analysis of fNIRS data: a comprehensive review," NeuroImage 85, 72-91 (2014).

193. J. Virtanen, T. Noponen, and P. Meriläinen, "Comparison of principal and independent component analysis in removing extracerebral interference from near-infrared spectroscopy signals," J. Biomed. Opt. 14(5), 054032 (2009).

194. X. Zhang, J. A. Noah, and J. Hirsch, "Separation of the global and local components in functional near-infrared spectroscopy signals using principal component spatial filtering," Neurophotonics 3(1), 015004 (2016)

195. G. Bauernfeind et al., "Separating heart and brain: on the reduction of physiological noise from multichannel functional near-infrared spectroscopy (fNIRS) signals," J. Neural Eng. 11(5), 056010 (2014).
196. F. B. Haeussinger et al., "Reconstructing functional near-infrared spectroscopy (fNIRS) signals impaired by extra-cranial confounds: an easy-to-use filter method," NeuroImage 95, 69-79 (2014).

197. A. R. Harrivel et al., "Dynamic filtering improves attentional state prediction with fNIRS," Biomed. Opt. Express 7(3), 979-1002 (2016).

198. R. B. Saager and A. J. Berger, "Direct characterization and removal of interfering absorption trends in two-layer turbid media," J. Opt. Soc. Am. A 22(9), 1874-1882 (2005).

199. E. Kirilina et al., "The physiological origin of task-evoked systemic artefacts in functional near infrared spectroscopy," Neurolmage 61(1), 70-81 (2012).

200. F. Scholkmann, A. J. Metz, and M. Wolf, "Measuring tissue hemodynamics and oxygenation by continuous-wave functional nearinfrared spectroscopy-how robust are the different calculation methods against movement artifacts?" Physiol. Meas. 35(4), 717-734 (2014).

201. R. Saager and A. Berger, "Measurement of layer-like hemodynamic trends in scalp and cortex: implications for physiological baseline suppression in functional near-infrared spectroscopy," J. Biomed. Opt. 13(3), 034017 (2008).

202. L. Gagnon et al., "Short separation channel location impacts the performance of short channel regression in NIRS," NeuroImage 59(3), 2518-2528 (2012).

203. L. Gagnon et al., "Improved recovery of the hemodynamic response in diffuse optical imaging using short optode separations and state-space modeling," NeuroImage 56(3), 1362-1371 (2011).

204. L. Gagnon et al., "Further improvement in reducing superficial contamination in NIRS using double short separation measurements," Neurolmage 85(Pt 1), 127-135 (2014).

205. J. R. Goodwin, C. R. Gaudet, and A. J. Berger, "Short-channel functional near-infrared spectroscopy regressions improve when sourcedetector separation is reduced," Neurophotonics 1(1), 015002 (2014).

206. T. Sato et al., "Reduction of global interference of scalp-hemodynamics in functional near-infrared spectroscopy using short distance probes," NeuroImage 141, 120-132 (2016).

207. Y. Zhang et al., "Multiregional functional near-infrared spectroscopy reveals globally symmetrical and frequency-specific patterns of superficial interference," Biomed. Opt. Express 6(8), 2786-2802 (2015).

208. R. B. Saager, N. L. Telleri, and A. J. Berger, "Two-detector corrected near infrared spectroscopy (C-NIRS) detects hemodynamic activation responses more robustly than single-detector NIRS," NeuroImage 55(4), 1679-1685 (2011).

209. T. Miyazawa et al., "Skin blood flow influences cerebral oxygenation measured by near-infrared spectroscopy during dynamic exercise," Eur. J. Appl. Physiol. 113(11), 2841-2848 (2013).

210. T. Takahashi et al., "Influence of skin blood flow on near-infrared spectroscopy signals measured on the forehead during a verbal fluency task," NeuroImage 57(3), 991-1002 (2011).

211. L. Gagnon et al., "Quantification of the cortical contribution to the NIRS signal over the motor cortex using concurrent NIRS-fMRI measurements," NeuroImage 59(4), 3933-3940 (2012).

212. P. G. Al-Rawi, P. Smielewski, and P. J. Kirkpatrick, "Evaluation of a near-infrared spectrometer (NIRO 300) for the detection of intracranial oxygenation changes in the adult head," Stroke 32(11), 2492-2500 (2001).

213. D. Canova et al., "Inconsistent detection of changes in cerebral blood volume by near infrared spectroscopy in standard clinical tests," J. Appl. Physiol. 110(6), 1646-1655 (2011).

214. A. Vrana et al., "Different mechanosensory stimulations of the lower back elicit specific changes in hemodynamics and oxygenation in cortical sensorimotor areas: a fNIRS study," Brain Behav. 6(12), e00575 (2016).

215. J. C. Ye et al., "NIRS-SPM: statistical parametric mapping for nearinfrared spectroscopy," NeuroImage 44(2), 428-447 (2009).

216. M. M. Plichta et al., "Model-based analysis of rapid event-related functional near-infrared spectroscopy (NIRS) data: a parametric validation study," NeuroImage 35(2), 625-634 (2007).

217. T. W. Wilson, M. J. Kurz, and D. J. Arpin, "Functional specialization within the supplementary motor area: a fNIRS study of bimanual coordination," Neurolmage 85, 445-450 (2014).

218. A. K. Singh et al., "Scope of resampling-based tests in fNIRS neuroimaging data analysis," Stat. Sin. 18, 1519-1534 (2008). 
219. M. W. Fagerland, "t-tests, non-parametric tests, and large studies-a paradox of statistical practice?" BMC Med. Res. Method. 12, 78 (2012).

220. S. Burke, "Missing values, outliers, robust statistics and non-parametric methods," LC-GC Eur. Online Suppl. Stat. Data Anal. 2, 19-24 (2001).

221. C. Potvin and D. A. Roff, "Distribution-free and robust statistical methods: viable alternatives to parametric statistics," Ecology 74(6), 1617-1628 (1993).

222. D. M. Erceg-Hurn and V. M. Mirosevich, "Modern robust statistical methods: an easy way to maximize the accuracy and power of your research," Am. Psychol. 63(7), 591-601 (2008).

223. M. P. Boisgontier and B. Cheval, "The ANOVA to mixed model transition," Neurosci. Biobehav. Rev. 68, 1004-1005 (2016).

224. E. Aarts et al., "A solution to dependency: using multilevel analysis to accommodate nested data," Nat. Neurosci. 17(4), 491-496 (2014).

225. C. M. Judd, J. Westfall, and D. A. Kenny, "Treating stimuli as a random factor in social psychology: a new and comprehensive solution to a pervasive but largely ignored problem," J. Pers. Social Psychol. 103(1), 54-69 (2012).

226. B. M. Bolker et al., "Generalized linear mixed models: a practical guide for ecology and evolution," Trends Ecol. Evol. 24(3), 127-135 (2009).

227. M. A. Kamran, M. M. N. Mannan, and M. Y. Jeong, "Cortical signal analysis and advances in functional near-infrared spectroscopy signal: a review," Front. Hum. Neurosci. 10, 261 (2016).

228. Y. Hoshi, "Functional near-infrared optical imaging: utility and limitations in human brain mapping," Psychophysiology 40(4), 511-520 (2003).

229. Y. Hoshi, N. Kobayashi, and M. Tamura, "Interpretation of near-infrared spectroscopy signals: a study with a newly developed perfused rat brain model," J. Appl. Physiol. 90(5), 1657-1662 (2001).

230. U. Lindauer et al., "Pathophysiological interference with neurovascular coupling - when imaging based on hemoglobin might go blind," Front. Neuroenerg. 2, 25 (2010).

231. A. Kleinschmidt et al., "Simultaneous recording of cerebral blood oxygenation changes during human brain activation by magnetic resonance imaging and near-infrared spectroscopy," J. Cereb. Blood Flow Metab. 16(5), 817-826 (1996).

232. V. Toronov et al., "Investigation of human brain hemodynamics by simultaneous near-infrared spectroscopy and functional magnetic resonance imaging," Med. Phys. 28(4), 521-527 (2001).

233. C. La Fougère et al., "Real versus imagined locomotion: a $\left[{ }^{18} \mathrm{~F}\right]-\mathrm{FDG}$ PET-fMRI comparison," Neurolmage 50(4), 1589-1598 (2010).

234. A. Zwergal et al., "Aging of human supraspinal locomotor and postural control in fMRI," Neurobiol. Aging 33(6), 1073-1084 (2012).

235. A. Zwergal et al., "Functional disturbance of the locomotor network in progressive supranuclear palsy," Neurology 80(7), 634-641 (2013).

236. J. G. Nutt, F. B. Horak, and B. R. Bloem, "Milestones in gait, balance, and falling," Mov. Disord. 26(6), 1166-1174 (2011).

237. A. Maillet, P. Pollak, and B. Debu, "Imaging gait disorders in parkinsonism: a review," J. Neurol. Neurosurg. Psychiatry 83(10), 986-993 (2012).

238. D. J. Clark, "Automaticity of walking: functional significance, mechanisms, measurement and rehabilitation strategies," Front. Hum. Neurosci. 9, 246 (2015).

239. P. M. M. Young and J. B. Dingwell, "Voluntary changes in step width and step length during human walking affect dynamic margins of stability," Gait Posture 36(2), 219-224 (2012).

240. E. Nordin et al., "Changes in step-width during dual-task walking predicts falls," Gait Posture 32(1), 92-97 (2010).

241. G. Rizzolatti, L. Fogassi, and V. Gallese, "Motor and cognitive functions of the ventral premotor cortex," Curr. Opin. Neurobiol. 12(2), 149-154 (2002).

242. R. I. Schubotz and D. von Cramon, "Functional-anatomical concepts of human premotor cortex: evidence from fMRI and PET studies," NeuroImage 20, S120-S131 (2003).

243. O. A. van den Heuvel et al., "Frontostriatal system in planning complexity: a parametric functional magnetic resonance version of tower of London task," NeuroImage 18(2), 367-374 (2003).
244. G. Cona and C. Semenza, "Supplementary motor area as key structure for domain-general sequence processing: a unified account," Neurosci. Biobehav. Rev. 72, 28-42 (2017).

245. J. R. Tregellas, D. B. Davalos, and D. C. Rojas, "Effect of task difficulty on the functional anatomy of temporal processing," NeuroImage 32(1), 307-315 (2006).

246. P. Nachev, C. Kennard, and M. Husain, "Functional role of the supplementary and pre-supplementary motor areas," Nat. Rev. Neurosci. 9(11), 856-869 (2008).

247. A. Dietrich, "Transient hypofrontality as a mechanism for the psychological effects of exercise," Psychiatry Res. 145(1), 79-83 (2006).

248. V. E. Kelly, A. J. Eusterbrock, and A. Shumway-Cook, "Factors influencing dynamic prioritization during dual-task walking in healthy young adults," Gait Posture 37(1), 131-134 (2013).

249. G. Yogev-Seligmann, J. M. Hausdorff, and N. Giladi, "Do we always prioritize balance when walking? Towards an integrated model of task prioritization," Mov. Disord. 27(6), 765-770 (2012).

250. D. Carius et al., "Hemodynamic response alteration as a function of task complexity and expertise-an fNIRS study in jugglers," Front. Hum. Neurosci. 10, 126 (2016).

251. E. Wittenberg et al., "Neuroimaging of human balance control: a systematic review," Front. Hum. Neurosci. 11, 170 (2017).

252. T. Brandt et al., "Reciprocal inhibitory visual-vestibular interaction: visual motion stimulation deactivates the parieto-insular vestibular cortex," Brain: J. Neurol. 121(Pt 9), 1749-1758 (1998).

253. K. Jahn et al., "Brain activation patterns during imagined stance and locomotion in functional magnetic resonance imaging," NeuroImage 22(4), 1722-1731 (2004).

254. S. Bense et al., "Multisensory cortical signal increases and decreases during vestibular galvanic stimulation (fMRI), J. Neurophysiol. 85(2), 886-899 (2001).

255. H. O. Karnath, "New insights into the functions of the superior temporal cortex," Nat. Rev. Neurosci. 2(8), 568-576 (2001).

256. A. Faraldo-García et al., "Influence of gender on the sensory organisation test and the limits of stability in healthy subjects," Acta Otorrinolaringol. 62(5), 333-338 (2011).

257. A. Dietrich, "Imaging the imagination: the trouble with motor imagery," Methods 45(4), 319-324 (2008).

258. J. L. Nasar and D. Troyer, "Pedestrian injuries due to mobile phone use in public places," Accid. Anal. Prev. 57, 91-95 (2013).

259. D. Stavrinos, K. W. Byington, and D. C. Schwebel, "Distracted walking: cell phones increase injury risk for college pedestrians," J. Saf. Res. 42(2), 101-107 (2011).

260. D. Hamacher et al., "The reliability of local dynamic stability in walking while texting and performing an arithmetical problem," Gait Posture 44, 200-203 (2016).

261. R. Cabeza, "Hemispheric asymmetry reduction in older adults: the HAROLD model," Psychol. Aging 17(1), 85-100 (2002).

262. S. Heuninckx, N. Wenderoth, and S. P. Swinnen, "Systems neuroplasticity in the aging brain: recruiting additional neural resources for successful motor performance in elderly persons," J. Neurosci. 28(1), 91-99 (2008).

263. D. A. E. Bolton, "The role of the cerebral cortex in postural responses to externally induced perturbations," Neurosci. Biobehav. Rev. 57, 142-155 (2015).

264. V. M. Clark and A. M. Burden, "A 4-week wobble board exercise programme improved muscle onset latency and perceived stability in individuals with a functionally unstable ankle," Phys. Ther. Sport 6(4), 181-187 (2005).

265. J. U. Wester et al., "Wobble board training after partial sprains of the lateral ligaments of the ankle: a prospective randomized study," J. Orthop. Sports Phys. Ther. 23(5), 332-336 (1996).

266. A. T. Onigbinde, T. Awotidebe, and H. Awosika, "Effect of 6 weeks wobble board exercises on static and dynamic balance of stroke survivors," Technol. Health Care 17(5-6), 387-392 (2009).

267. D. Hamacher, D. Hamacher, and L. Schega, "Towards the importance of minimum toe clearance in level ground walking in a healthy elderly population," Gait Posture 40(4), 727-729 (2014).

268. R. Morris et al., "Gait and cognition: mapping the global and discrete relationships in ageing and neurodegenerative disease," Neurosci. Biobehav. Rev. 64, 326-345 (2016). 
Fabian Herold received his BA degree in sport science from Otto von Guericke University in 2014. Currently, he is working in the Department of Sport Science at Otto von Guericke University as a research assistant, where he finished his MA thesis. His research interests include analyzing neuromotor control processes of gait and the application of functional near-infrared spectroscopy in motor control experiments.

Patrick Wiegel received his BA degree from Otto von Guericke University Magdeburg in 2014 and his MSc degree from AlbertLudwigs-University Freiburg in 2016. Currently, he is a PhD student at Albert-Ludwigs-University Freiburg. His research focuses on the underlying neural processes of human motor control and motor learning.

Felix Scholkmann received his $\mathrm{PhD}$ at the University of Zurich, Switzerland, in 2014. As a postdoc at the Biomedical Optics Research Laboratory of the University Hospital Zurich and a research associate at the University of Bern, his research focuses on biomedical signal processing, biomedical optics (development and application of fNIRS for human optical neuroimaging), neuroscience, integrative physiology, and biophysics.
Angelina Thiers received her master's degree in information technology from the University of Applied Science in Brandenburg in 2014 She is a researcher at the Chair Health and Physical Activity at Otto von Guericke University Magdeburg, Germany. Her research interests include biosignal analyses and the development of new applications.

Dennis Hamacher received his doctorate from Otto von Guericke University in 2017. At the Chair Health and Physical Activity, he is working as a research associate, where he analyzes the underlying mechanisms of gait stability in old and diseased cohorts.

Lutz Schega is a professor (full) and chair of the Department of Health and Physical Activity at Otto von Guericke University in Magdeburg. He received his $\mathrm{PhD}$ in sports science in 1994 and his habilitation (postdoctoral qualification) in sports and rehabilitation science in 2003 from the University of Leipzig. A major focus of his work is on investigating various aspects of human walking performance. 